\title{
Computational vascular fluid-structure interaction: methodology and application to cerebral aneurysms
}

\author{
Y. Bazilevs - M.-C. Hsu • Y. Zhang - W. Wang • \\ T. Kvamsdal • S. Hentschel · J. G. Isaksen
}

Received: 13 April 2009 / Accepted: 11 January 2010 / Published online: 29 January 2010

(C) The Author(s) 2010. This article is published with open access at Springerlink.com

\begin{abstract}
A computational vascular fluid-structure interaction framework for the simulation of patient-specific cerebral aneurysm configurations is presented. A new approach for the computation of the blood vessel tissue prestress is also described. Simulations of four patient-specific models are carried out, and quantities of hemodynamic interest such as wall shear stress and wall tension are studied to examine the relevance of fluid-structure interaction modeling when compared to the rigid arterial wall assumption. We demonstrate that flexible wall modeling plays an important role in accurate prediction of patient-specific hemodynamics. Discussion of the clinical relevance of our methods and results is provided.
\end{abstract}

Y. Bazilevs $(\bowtie) \cdot$ M.-C. Hsu

Department of Structural Engineering, University of California, San Diego, 9500 Gilman Drive, La Jolla, CA 92093, USA e-mail: yuri@ucsd.edu

\section{Y. Zhang $\cdot$ W. Wang}

Department of Mechanical Engineering, Carnegie Mellon

University, Pittsburgh, PA 15213, USA

\section{T. Kvamsdal}

Department of Applied Mathematics, SINTEF Information and Communication Technology,

7465 Trondheim, Norway

\section{S. Hentschel}

Department of Scientific Computing, Simula,

Martin Linges vei 17, 1364 Fornebu, Norway

J. G. Isaksen

Departments of Neurosurgery and Neurology, University Hospital of North Norway, 9038 Troms $\emptyset$, Norway

J. G. Isaksen Institute of Clinical Medicine, University of Troms $\varnothing$, 9037 Troms $\varnothing$, Norway
Keywords Cerebral aneurysms $\cdot$ Fluid-structure interaction - Arterial wall tissue modeling - Incompressible Navier-Stokes equations · Boundary layer meshing · Wall shear stress - Wall tension - Tissue prestress

\section{Introduction}

Starting with a pioneering work on patient-specific vascular modeling in Taylor et al. (1998), the field of computational vascular and cardiovascular modeling has matured immensely over the last decade. Numerous advances in the simulation technology were proposed, such as imposition of physiologically-realistic outflow boundary conditions (Formaggia et al. 2001; Lagana et al. 2002; VignonClementel et al. 2006), simulation of stenting technology in the context of cerebral aneurysms (Appanaboyina et al. 2009) and coronary arteries (Zunino et al. 2009), optimization of cardiovascular geometries for surgical treatment (Marsden et al. 2008), inclusion of the effects of wall elasticity (Figueroa et al. 2006; Bazilevs et al. 2006, 2008; Tezduyar et al. 2007; Torii et al. 2008, 2009), and growth and remodeling (Figueroa et al. 2009) in the simulations. Nowadays, the state-of-the-art in computational hemodynamics involves fully coupled fluid-structure patient-specific simulations of large portions of the human cardiovascular system. Simulations are performed in an effort to investigate hemodynamic factors influencing the onset and progression of cardiovascular disease, to predict an outcome of a surgical intervention, or to evaluate the effects of electromechanical assist devices.

Currently, assessment of aneurysm rupture risk is based on known risk factors like smoking, hypertension, family history of subarachnoid hemorrhage, and aneurysm size, derived from epidemiological and clinical studies. However, the current knowledge is not sufficient for patient-specific clinical 
decision-making. Recently, the aneurysm shape was proposed as an important, patient-specific, independent rupture risk factor (Aneurysm Registry of Helsinki 2009; Aneurysm Registry of Troms $\varnothing 2009$; Frösen J. Private communication 2009). As a result, in recent years, a considerable effort was put forth to apply pure computational fluid dynamics (CFD) techniques to study and classify flow patterns and wall shear stress (WSS) and oscillatory shear index (OSI) distributions in a large sample of patient-specific cerebral aneurysm shapes (see, e.g., Sforza et al. 2009 and references therein). These quantities of hemodynamic interest, practically unattainable in experiments or measurements, are connected to clinical events, which help better understand the disease processes and improve patient evaluation and treatment.

When pure CFD is used for vascular blood flow simulations, it is assumed that the vessel wall remains rigid. The rigid wall assumption does not properly reflect the behavior of real blood vessels that deform under the action of blood flow forces and, in turn, alter the details of blood flow. For the modeling to be realistic, coupled fluidstructure interaction (FSI) modeling must be employed. However, high-fidelity FSI of vascular blood flow in a patient-specific setting is scarce, which is mainly due to the numerical challenges involved. Despite its modeling shortcomings, pure CFD remains the predominant modeling approach for vascular blood flow, and, in particular, for computation of aneurysm flows. Notable exceptions include the work of (Takizawa et al. 2010a,b; Torii et al. 2006a,b, 2007, 2008,2009 ) on cerebral aneurysms and the work of (Wolters et al. 2005; Scotti and Finol 2007; Rissland et al. 2009) on aortic abdominal aneurysms. This paper likewise focuses on developing and using advanced FSI computational techniques to assess the risk of rupture for cerebral aneurysms in individual patients. Our FSI framework involves the coupling of incompressible fluid representing the blood and a hyperelastic solid representing vessel wall tissue. The equations are posed on a moving domain and are equipped with an appropriate set of boundary and initial conditions. Variable wall thickness, tissue prestress, sliding inlet and outlet branch boundary conditions, and boundary layer meshing are the key ingredients of our computational framework that allow for physiologically realistic and accurate simulations. It should also be noted that the importance of the problem of cerebral aneurysm rupture and its resolution by means of advanced numerical simulation was discussed in a recent review article on open problem in vascular modeling (Taylor and Humphrey 2009).

This paper is outlined as follows. In Sect. 2, we recall the formulation of the fluid and solid sub-problems and their interface conditions that ensure appropriate coupling between the two systems. The basic coupled formulation was developed in Bazilevs et al. (2008) and the modeling is further improved here. We introduce the vessel wall tissue prestress that was not accounted for in our original modeling framework. The need for modeling tissue prestress arises due to the fact that the arterial configuration coming from patient-specific imaging data is subjected to intramural blood pressure and viscous forces. As a result, it may not be taken as a stress-free reference configuration. An approach is presented that circumvents this difficulty by prescribing a state of prestress to the arterial tissue that puts the artery in equilibrium with the blood flow forces. In Sect. 3, we briefly recall our meshing and basic computational procedures for the simulation of arterial fluid-structure interaction phenomena. Patient-specific models are presented, and mesh statistics are summarized. Out of the four patient-specific aneurysm models analyzed, two correspond to ruptured and two to unruptured cases. Fine meshes with boundary layer resolution are employed, which ensures high fidelity of the computational results. Simulations are driven by a prescribed time-periodic inlet velocity and outlet resistance boundary conditions that ensure physiological pressure levels in the vessels. We also introduce free-slip boundary conditions at the model inlets and outlets. This gives the inlet and outlet branches a flexibility to move in their cut planes as well as deform radially in response to variations in the intramural pressure and viscous forces. Free-slip boundary conditions lead to more realistic vessel wall deformations than fixed inlets and outlets that were employed previously. In Sect. 4, we present our simulation results, focusing on the comparison between rigid and flexible simulations. While the differences in the computed blood flow speeds are not as significant (although clearly visible in some cases), the wall shear stress was found to be consistently overestimated in the rigid wall simulations, in one case by as much as $52 \%$, which is felt to be a significant overestimation. In Sect. 5, we draw conclusions and provide discussion of the clinical relevance of our findings.

\section{Continuum modeling}

\subsection{Blood flow modeling}

The blood flow is governed by the Navier-Stokes equations of incompressible flow posed on a moving domain. The Arbitrary Lagrangian-Eulerian (ALE) formulation is used, which is a widely used approach for vascular blood flow applications (Nobile 2001; Formaggia et al. 2001; Gerbeau et al. 2005; Fernández et al. 2008). Alternatively, one can apply the space-time methodology (Tezduyar et al. 2007; Torii et al. 2008, 2009), which leads to better time accuracy, yet somewhat higher computational expense per time step.

Let $\mathcal{V}_{f}$ and $\mathcal{W}_{f}$ be the standard solution and weighting function spaces for the fluid problem. The variational formulation of the Navier-Stokes equations is stated as follows: 
Fig. 1 Dimension, reference geometry and meshes for Models 1-4 of the middle cerebral artery (MCA) bifurcation. Inlet branches are labeled M1 and outlet branches are labeled M2. The arrows point in the direction of inflow velocity

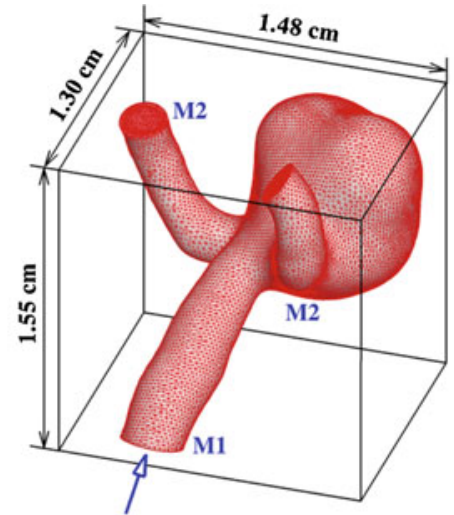

(a) Model 1

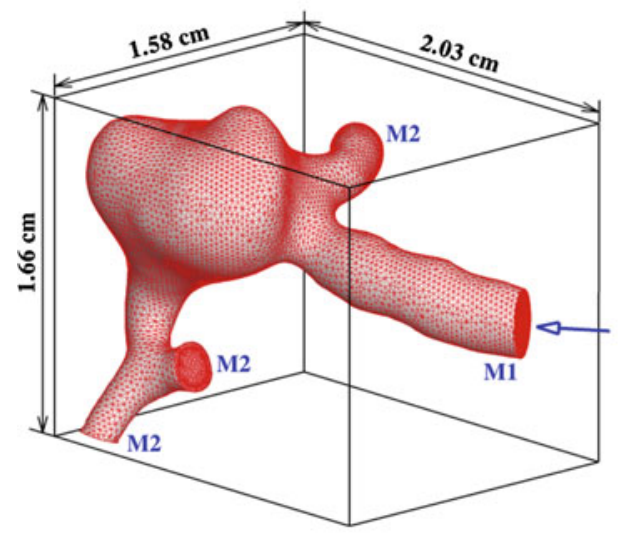

(c) Model 3

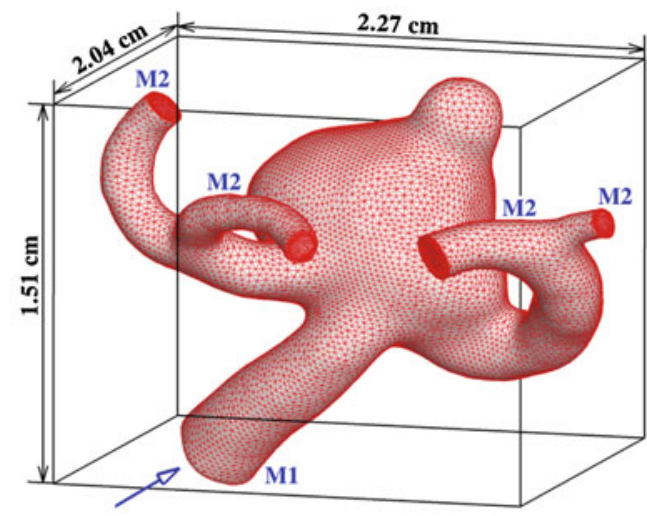

(b) Model 2

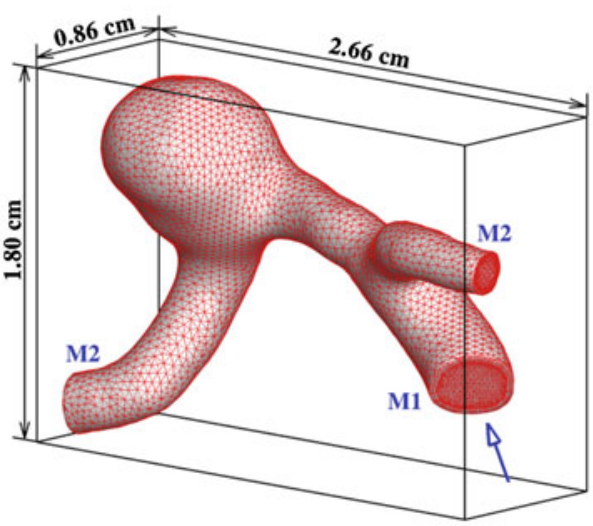

(d) Model 4

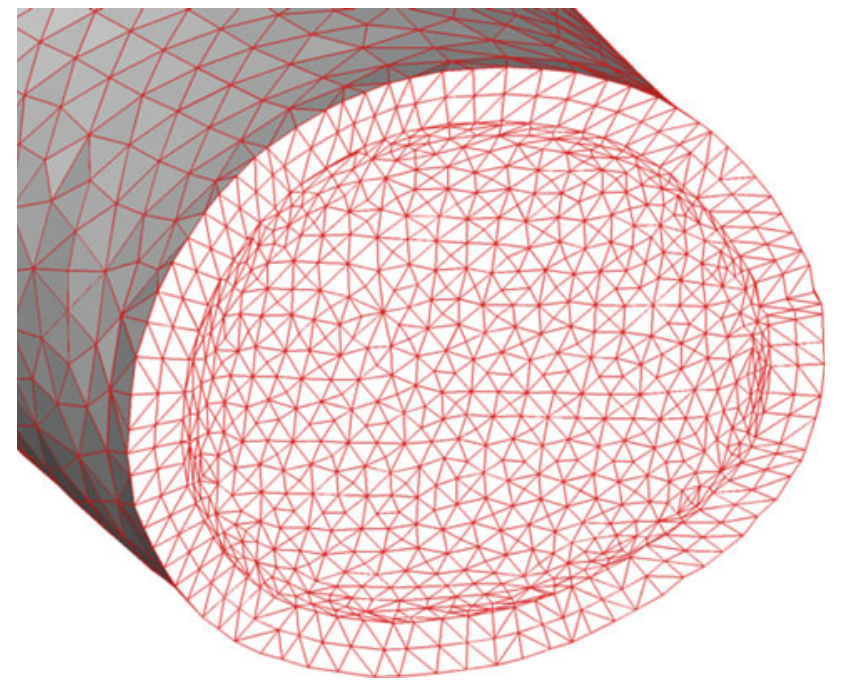

Fig. 2 Zoom on the mesh of the inlet surface of Model 1. Solid and boundary layer fluid mesh are shown

Find the velocity-pressure pair $\{\boldsymbol{v}, p\} \in \mathcal{V}_{f}$, such that for all weighting functions $\{\boldsymbol{w}, q\} \in \mathcal{W}_{f}$,

$$
\begin{gathered}
\left(\boldsymbol{w}, \rho \frac{\partial \boldsymbol{v}}{\partial t}\right)_{\Omega_{t}}+(\boldsymbol{w}, \rho(\boldsymbol{v}-\hat{\boldsymbol{v}}))_{\Omega_{t}}+\left(q, \nabla_{x} \cdot \boldsymbol{v}\right)_{\Omega_{t}} \\
+\left(\nabla_{x}^{s} \boldsymbol{w}, \boldsymbol{\sigma}^{f}\right)_{\Omega_{t}}=(\boldsymbol{w}, \rho \boldsymbol{f})_{\Omega_{t}}+(\boldsymbol{w}, \boldsymbol{h})_{\Gamma_{t}^{N}}
\end{gathered}
$$

where $\hat{v}$ is the velocity of the fluid domain, $\nabla_{x}$ is the gradient operator on $\Omega_{t}, \nabla_{x}^{s}$ is its symmetrization, $\Gamma_{t}^{N}$ is the Neumann part of the fluid domain boundary, $\boldsymbol{h}$ is the boundary traction vector, $\boldsymbol{f}$ is the body force per unit mass, $\rho$ is the density of the fluid, and $(\cdot, \cdot)_{A}$ denotes the usual $L^{2}$-inner product over $A$. The variational Eq. (1) represents the balance of mass and linear momentum for the incompressible fluid.

The true or Cauchy stress $\sigma^{f}$ for the incompressible Newtonian fluid is given through a constitutive law that holds on the spatial domain as

$\boldsymbol{\sigma}^{f}=-p \boldsymbol{I}+2 \mu^{f} \nabla_{x}^{s} \boldsymbol{v}$,

where $p$ is the fluid pressure and $\mu^{f}$ is the dynamic viscosity. In this work, the blood is modeled as a Newtonian fluid. Although the blood is generally considered to be a nonNewtonian fluid, it was shown in Cebral et al. (2005) that the Newtonian assumption is sufficient for cerebral aneurysm flows.

\subsection{Arterial tissue modeling}

\subsubsection{Kinematics and constitutive modeling}

Let $\boldsymbol{X}$ be the coordinates of the initial or reference configuration, and let $\boldsymbol{u}$ be the displacement with respect to the 
Table 1 Finite element mesh sizes for the aneurysm models

\begin{tabular}{lllll}
\hline Model & Fluid elements & Solid elements & Total elements & Total nodes \\
\hline 1 & 407,280 & 120,000 & 527,280 & 94,199 \\
2 & 341,813 & 120,684 & 462,497 & 83,591 \\
3 & 232,652 & 83,598 & 316,250 & 57,379 \\
4 & 96,684 & 47,928 & 144,612 & 26,947 \\
\hline
\end{tabular}

Table 2 Inflow cross-sectional areas for the aneurysm models

\begin{tabular}{ll}
\hline Model & Inflow surface area $\left(\mathrm{cm}^{2}\right)$ \\
\hline 1 & $4.2452 \times 10^{-2}$ \\
2 & $9.2985 \times 10^{-2}$ \\
3 & $5.6349 \times 10^{-2}$ \\
4 & $8.7916 \times 10^{-2}$ \\
\hline
\end{tabular}

initial configuration. Then, $\boldsymbol{x}$, the coordinates of the current configuration, is given by

$\boldsymbol{x}=\boldsymbol{X}+\boldsymbol{u}$

The deformation gradient tensor $\boldsymbol{F}$, the Cauchy-Green deformation tensor $\boldsymbol{C}$, and the Green-Lagrangian strain tensor $\boldsymbol{E}$, are defined as

$\boldsymbol{F}=\frac{\partial \boldsymbol{x}}{\partial \boldsymbol{X}}=\boldsymbol{I}+\frac{\partial \boldsymbol{u}}{\partial \boldsymbol{X}}$,

$\boldsymbol{C}=\boldsymbol{F}^{T} \boldsymbol{F}$,

$\boldsymbol{E}=\frac{1}{2}(\boldsymbol{C}-\boldsymbol{I})$,

respectively.

We model the arterial tissue as a three-dimensional hyperelastic solid and assume the existence of a stored elastic energy in the form

$\varphi(\boldsymbol{C}, J)=\frac{1}{2} \mu^{s}\left(J^{-2 / 3} \operatorname{tr} \boldsymbol{C}-3\right)+\frac{1}{2} \kappa^{s}\left(\frac{1}{2}\left(J^{2}-1\right)-\ln J\right)$.

In (7), $J=\operatorname{det} \boldsymbol{F}$, and $\mu^{s}$ and $\kappa^{s}$ are identified with the material shear and bulk moduli, respectively. From (7), the second Piola-Kirchhoff stress tensor $S$ and the fourth-rank tensor of material tangent moduli $\mathbf{C}$ are obtained as

$$
\begin{aligned}
\boldsymbol{S} & =2 \frac{\partial \varphi}{\partial \boldsymbol{C}}(\boldsymbol{C}, J) \\
& =\mu^{s} J^{-2 / 3}\left(\boldsymbol{I}-\frac{1}{3} \operatorname{tr} \boldsymbol{C} \boldsymbol{C}^{-1}\right)+\frac{1}{2} \kappa^{s}\left(J^{2}-1\right) \boldsymbol{C}^{-1},
\end{aligned}
$$

and

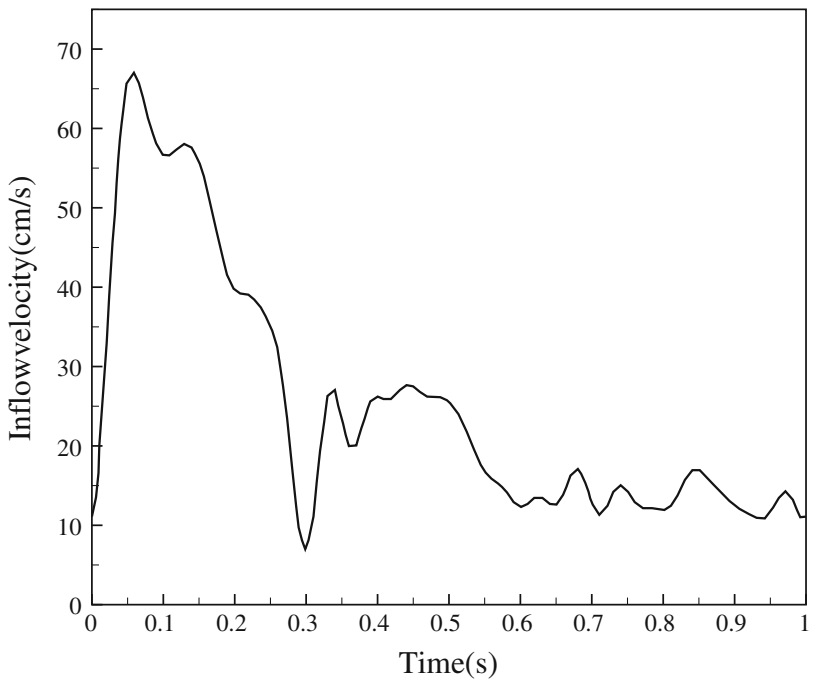

Fig. 3 Area-averaged inflow velocity as a function of time during the heart cycle. The inflow velocity profile is taken from Isaksen et al. (2008)

$$
\begin{aligned}
\mathbf{C}=4 & \frac{\partial^{2} \varphi}{\partial \boldsymbol{C} \partial \boldsymbol{C}}(\boldsymbol{C}, J) \\
= & \left(\frac{2}{9} \mu^{s} \boldsymbol{J}^{-2 / 3} \operatorname{tr} \boldsymbol{C}+\kappa^{s} J^{2}\right) \boldsymbol{C}^{-1} \otimes \boldsymbol{C}^{-1} \\
& +\left(\frac{2}{3} \mu J^{-2 / 3} \operatorname{tr} \boldsymbol{C}-\kappa^{s}\left(J^{2}-1\right)\right) \boldsymbol{C}^{-1} \odot \boldsymbol{C}^{-1} \\
& -\frac{2}{3} \mu^{s} J^{-2 / 3}\left(\boldsymbol{I} \otimes \boldsymbol{C}^{-1}+\boldsymbol{C}^{-1} \otimes \boldsymbol{I}\right) .
\end{aligned}
$$

In (10), the symbols $\otimes$ and $\odot$ are defined as

$\left(\boldsymbol{C}^{-1} \otimes \boldsymbol{C}^{-1}\right)_{I J K L}=\left(\boldsymbol{C}^{-1}\right)_{I J}\left(\boldsymbol{C}^{-1}\right)_{K L}$,

$\left(\boldsymbol{C}^{-1} \odot \boldsymbol{C}^{-1}\right)_{I J K L}=\frac{\left(\boldsymbol{C}^{-1}\right)_{I K}\left(\boldsymbol{C}^{-1}\right)_{J L}+\left(\boldsymbol{C}^{-1}\right)_{I L}\left(\boldsymbol{C}^{-1}\right)_{J K}}{2}$.

To conclude this section, a few remarks about the proposed solid model are given.

Remark When the reference and current configurations coincide, the expression for the tensor of material tangent moduli $\mathbf{C}$ is simplified to

$$
(\mathbf{C})_{I J K L}=\left(\kappa^{s}-\frac{2}{3} \mu^{s}\right) \delta_{I J} \delta_{K L}+\mu^{s}\left(\delta_{I K} \delta_{J L}+\delta_{I L} \delta_{J K}\right) \text {. }
$$

In the above expression, we recognize a tensor of elastic moduli for an isotropic, linearly elastic material and interpret $\mu^{s}$ and $\kappa^{s}$ as the material shear and bulk moduli, respectively.

Remark The material model is assumed to be isotropic, and the material properties (i.e., the bulk and shear moduli) are chosen from our previous works on cerebral aneurysms (see, e.g., Isaksen et al. 2008). This is an oversimplification, 
Fig. 4 Wall thickness distribution

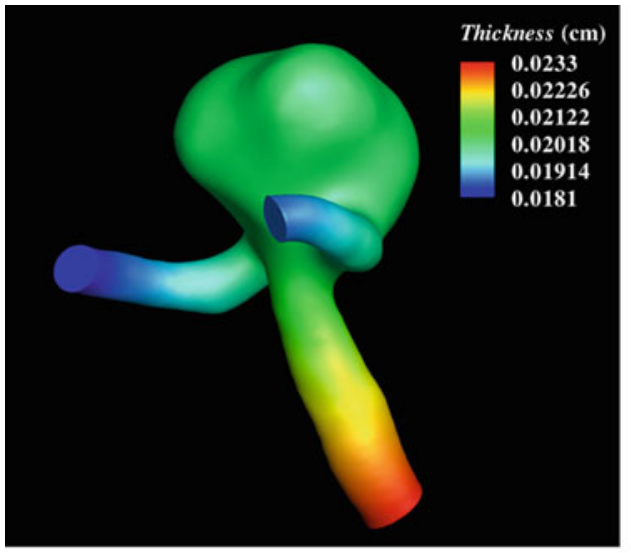

(a) Model 1

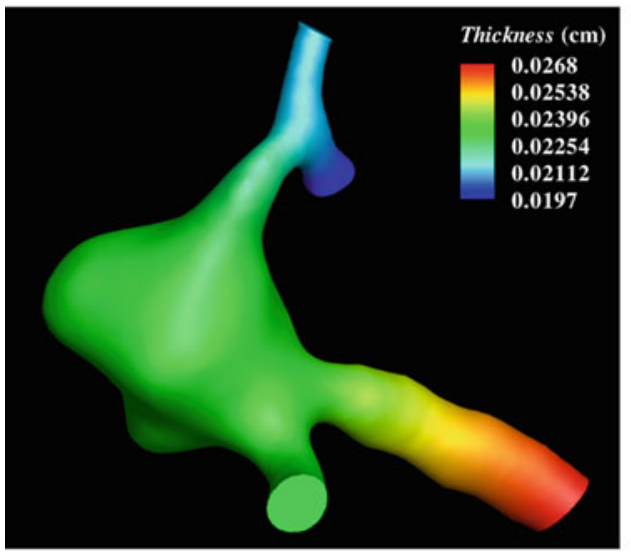

(c) Model 3

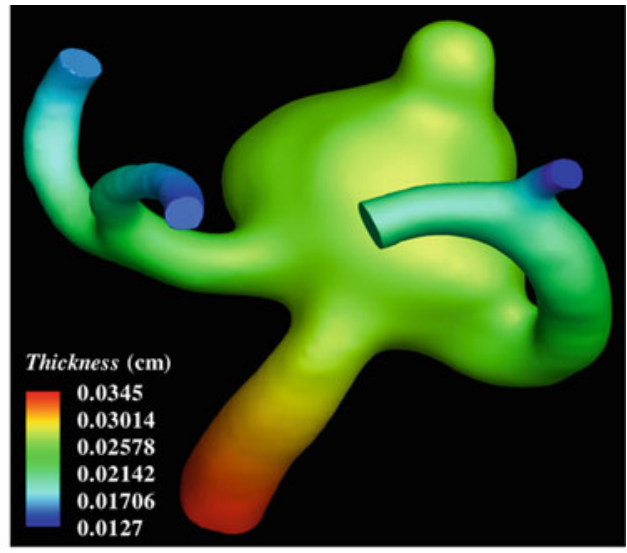

(b) Model 2

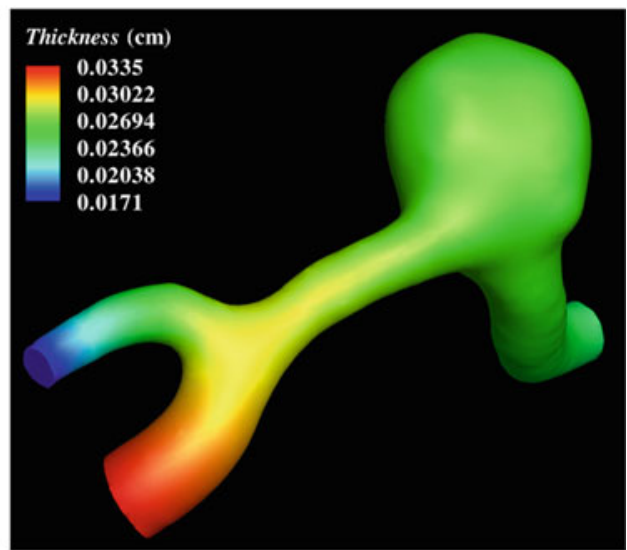

(d) Model 4 as it is known that the arterial wall tissue is composed of three distinct layers that are, in general, anisotropic. However, incorporating this information into three-dimensional patient-specific models is currently not feasible. Nevertheless, the authors feel that the solid model, together with the choice of the material parameters, provides reasonable inertial and stiffness properties of arterial tissue and is able to predict physiologically realistic wall deformation under the action of the fluid forces, as will evidenced by the numerical results presented in a later section.

Remark The material model originates from Simo and Hughes (1998), and its stress-strain behavior was analytically studied on simple cases of uniaxial strain (Bazilevs et al. 2008) and pure shear (Lipton et al. 2009). Mild stiffening with deformation was observed in both cases. Stiffening with deformation is a well-known characteristic of arterial tissue in the regime of large strains (see, e.g., Humphrey 2002). However, as we will see in the computations, the range of strains in the case of cerebral aneurysm fluid-structure interaction is such that the nature of the non-linearity is unlikely to be of great importance. As a result, and given the uncertainty in the material parameters and modeling errors associated with the choice of an isotropic material, a simple linear stress-strain relationship (i.e., the St. Venant-
Kirchhoff model) may have been adequate for the present application. However, despite its simplicity, we do not recommend using the St. Venant-Kirchhoff model because it is not well posed for the regime of strong compression (see, e.g., Holzapfel 2000), which may be present in the regions of arterial branching. The current model does not have this shortcoming. Unstable behavior for strong compression is precluded due to the presence of the $\ln J$ term in the strain energy function (7).

\subsubsection{Variational formulation of the solid problem}

Let $\mathcal{V}_{s}$ and $\mathcal{W}_{S}$ be the standard solution and weighting function spaces for the solid problem. The variational formulation of the solid problem is stated as follows: Find the displacement $\boldsymbol{u} \in \mathcal{V}_{s}$ such that for all weighting functions $\boldsymbol{w} \in \mathcal{W}_{s}$,

$$
\begin{aligned}
& \left(\boldsymbol{w}, \rho_{0} \frac{\partial^{2} \boldsymbol{u}}{\partial t^{2}}\right)_{\Omega_{0}}+\left(\nabla_{X} \boldsymbol{w}, \boldsymbol{F}\left(\boldsymbol{S}+\boldsymbol{S}_{0}\right)\right)_{\Omega_{0}} \\
& =\left(\boldsymbol{w}, \rho_{0} \boldsymbol{f}\right)_{\Omega_{0}}+(\boldsymbol{w}, \boldsymbol{h})_{\Gamma_{0}^{N}},
\end{aligned}
$$

where $\Omega_{0}$ is the solid domain in the reference configuration, $\Gamma_{0}^{N}$ is the Neumann part of the solid boundary, $\rho_{0}$ is the density of the solid in the reference configuration, $\boldsymbol{f}$ and $\boldsymbol{h}$ are 
Fig. 5 Tissue prestress

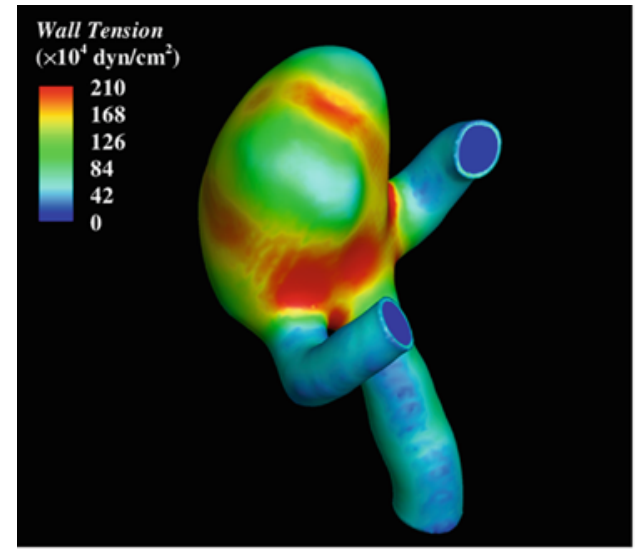

(a) Model 1

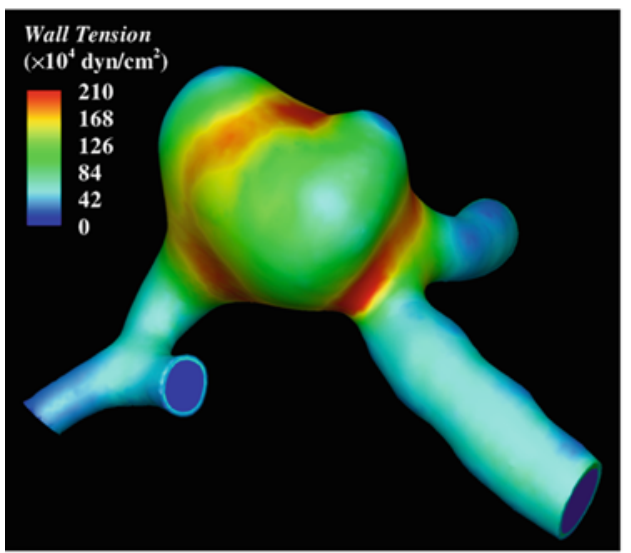

(c) Model 3

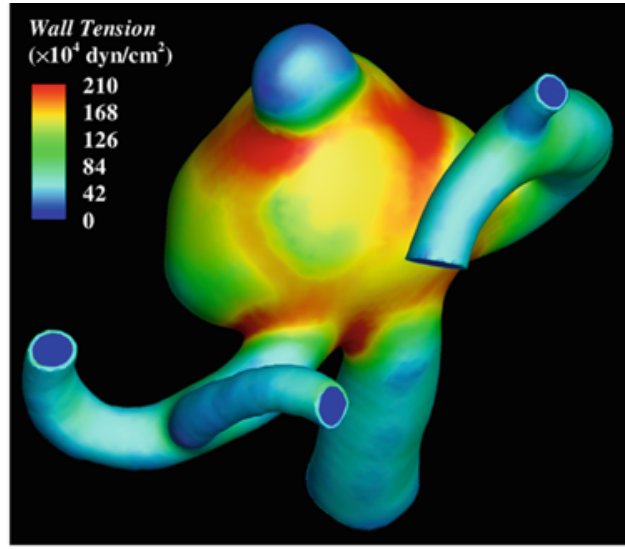

(b) Model 2

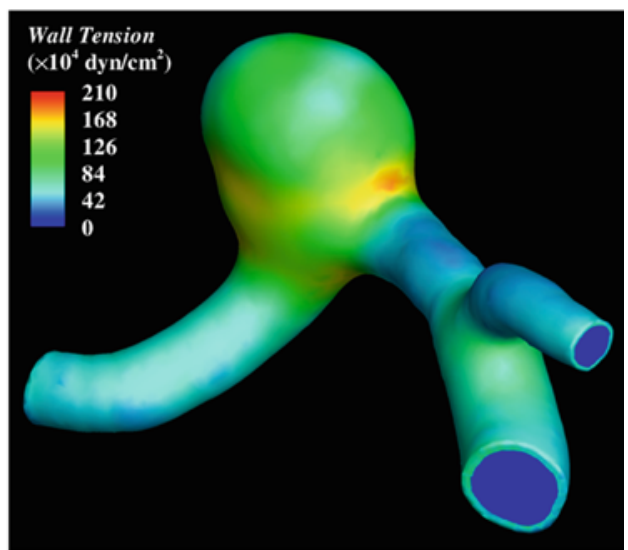

(d) Model 4 the body and surface forces, respectively. Variational Eq. (14) represents the balance of linear momentum for the solid.

This formulation is non-standard due to the presence of the $S_{0}$ in the stress term on the left-hand-side of (14). In our modeling framework, $\boldsymbol{S}_{0}$ is a prestress that is present in the artery reference configuration, which is assumed to coincide with the configuration extracted from patient-specific imaging data. This configuration is subjected to blood pressure and viscous forces and, in turn, develops internal stress to resist these loads. This internal stress tensor is denoted by $S_{0}$, and its computation is presented in the next section.

\subsubsection{Blood vessel tissue prestress}

The variational formulation of the prestress problem, which is posed over the same function spaces as the solid problem, is stated as follows: Find the displacement $\boldsymbol{u} \in \mathcal{V}_{s}$ such that $\forall \boldsymbol{w} \in \mathcal{W}_{S}$,

$\left(\nabla_{X} \boldsymbol{w}, \boldsymbol{F} \boldsymbol{S}\right)_{\Omega_{0}}+(\boldsymbol{w}, \boldsymbol{h})_{\Gamma_{0}^{f s}}=0$

where, $\Gamma_{0}^{f s}$ is the fluid-solid boundary in the reference configuration, $\boldsymbol{F}$ and $\boldsymbol{S}$ are defined in Eqs. (4) and (8), respec- tively, and $\boldsymbol{h}$ is the fluid traction vector consisting of both the pressure and viscous parts, given by

$\boldsymbol{h}=\boldsymbol{\sigma}^{f} \boldsymbol{n}^{f}$.

The fluid traction vector may be obtained from a rigid-wall blood flow simulation on a reference domain with steady inflow and resistance outflow boundary conditions. The latter guarantees a physiological intramural pressure level in the arteries. (For a discussion of outflow boundary conditions for cardiovascular simulations, see, e.g., Vignon-Clementel et al. 2006; Bazilevs et al. 2008, 2009a.)

The variational Eq. (15) are solved for the displacement field $\boldsymbol{u}$, and the corresponding stress field $\boldsymbol{S}$ is computed from the displacement field. The prestress $\boldsymbol{S}_{0}$ is assigned the value of $\boldsymbol{S}$, and the coupled time-dependent fluid-structural problem departs from the prestressed reference configuration with the initial displacement field set to zero.

Remark Because $\boldsymbol{h}$ is dominated by the intramural pressure part and the effect of the viscus forces is not as pronounced, one may simplify the definition of the fluid traction vector in (15) to

$\boldsymbol{h}=-\tilde{p}_{0} \boldsymbol{n}^{f}$, 
Fig. 6 Relative wall displacement between peak systole and low diastole

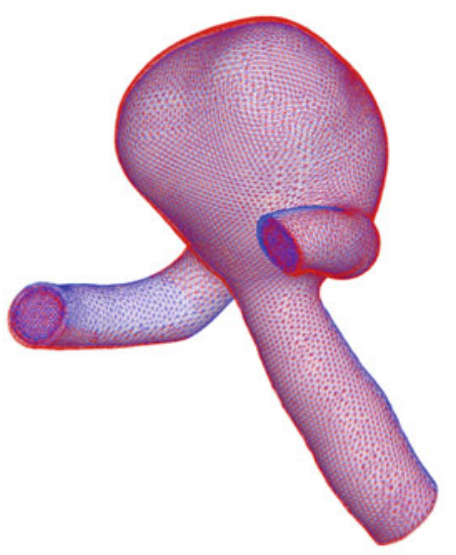

(a) Model 1

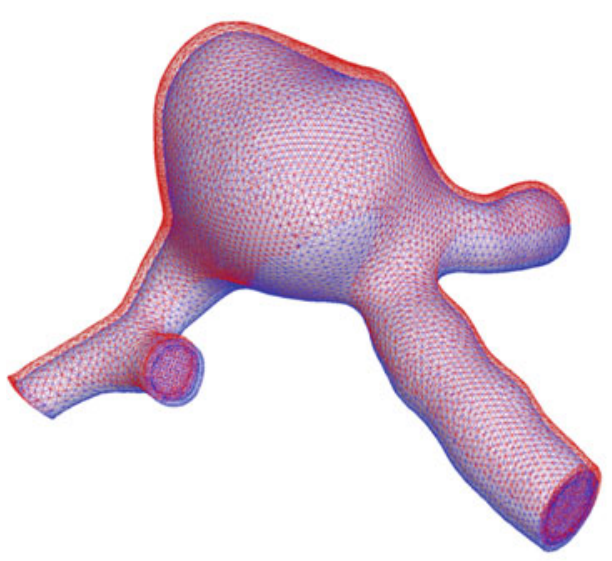

(c) Model 3

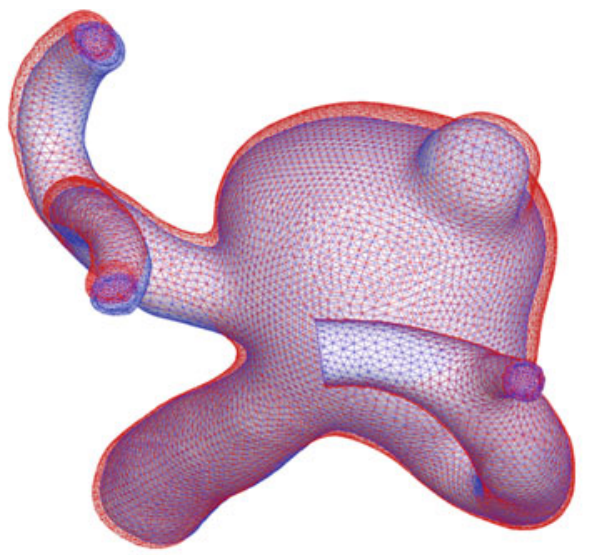

(b) Model 2

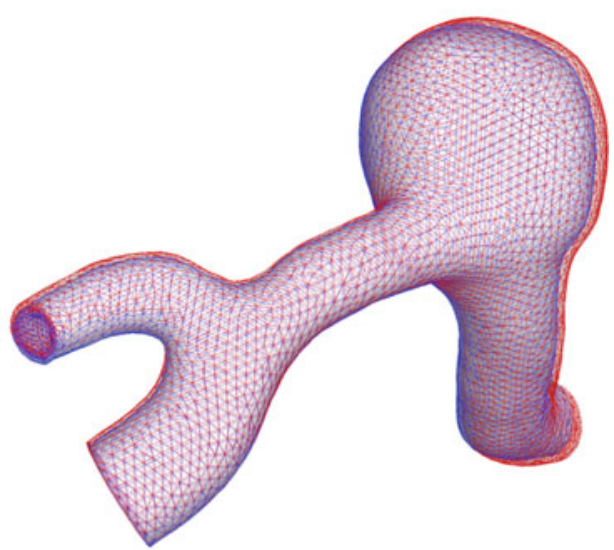

(d) Model 4 where $\tilde{p}_{0}$ is an averaged value of the intramural pressure over the cycle, which may be taken from patient data or literature. This approach, however, is not adopted in this work and a full fluid mechanics problem is solved to obtain the forcing for the prestress problem. Although the intramural pressure dominates the fluid traction vector, the effect of the viscous forces is small, yet non-negligible (see Tezduyar et al. 2009).

\subsection{Compatibility conditions at the fluid-solid interface}

No-slip boundary conditions hold at the fluid-solid interface. Traction compatibility conditions also hold at the fluid-solid interface, namely,

$\boldsymbol{\sigma}^{s} \boldsymbol{n}^{s}+\boldsymbol{\sigma}^{f} \boldsymbol{n}^{f}=\mathbf{0}$ on $\Gamma_{t}^{f s}$,

where $\boldsymbol{\sigma}^{s}$ and $\boldsymbol{\sigma}^{f}$ are the solid and fluid Cauchy stress tensors, $\boldsymbol{n}^{s}$ and $\boldsymbol{n}^{f}$ are the unit outward normal vectors to the solid and fluid subdomain boundaries, respectively, and $\Gamma^{f s}$ is the fluid-solid interface in the current configuration. The fluid Cauchy stress $\boldsymbol{\sigma}^{f}$ is evaluated directly according to Eq. (2), and the solid Cauchy stress $\sigma^{s}$ is computed as (see, e.g., Holzapfel 2000)
$\boldsymbol{\sigma}^{s}=J^{-1} \boldsymbol{F} \boldsymbol{S} \boldsymbol{F}^{T}$.

Equation (18) states that the fluid and solid forces are in equilibrium at the fluid-solid interface.

Because the solid undergoes motion under the influence of the fluid forces, the fluid-solid interface also displaces. This, in turn, imposes the displacement of the entire fluid region, which must also be modeled. We employ the equations of linear elasticity to model the fluid subdomain motion. These equations are posed on a time-dependent "nearby" configuration and are subjected to the displacement boundary conditions coming from the motion of the fluid-solid interface. This gives a well-defined current configuration of the fluid domain that conforms to the boundaries of the solid. In the discrete setting, this procedure ensures a smooth evolution of the computation mesh of the fluid domain. The nearby configuration typically corresponds to that of the previous time step in our computations (see, e.g., Bazilevs et al. 2008 for more details).

Remark In the case of the rigid wall assumption, the arterial wall is held fixed, in which case the blood flow model reduces to the incompressible Navier-Stokes equations posed on a 
Fig. 7 Principal in-plane strain nearly peak systole $(t=0.08 \mathrm{~s})$

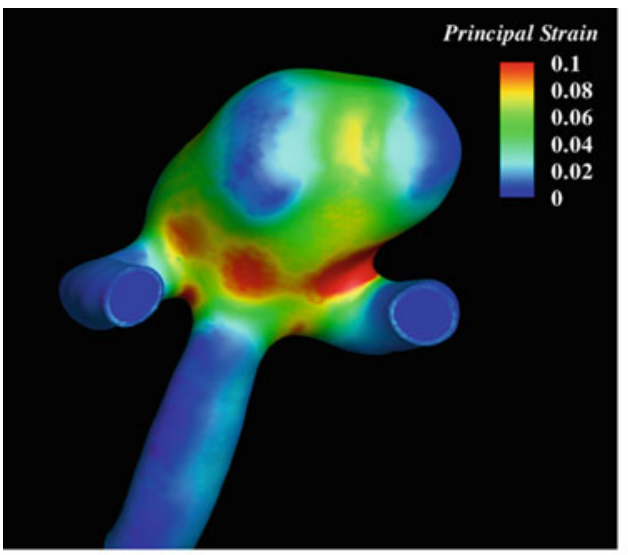

(a) Model 1

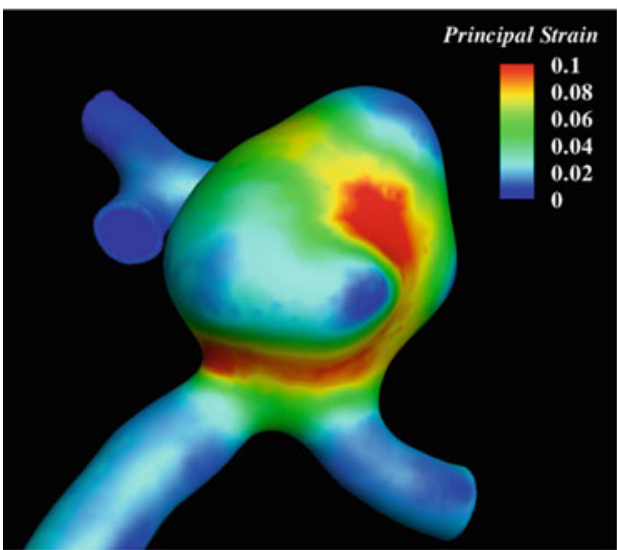

(c) Model 3

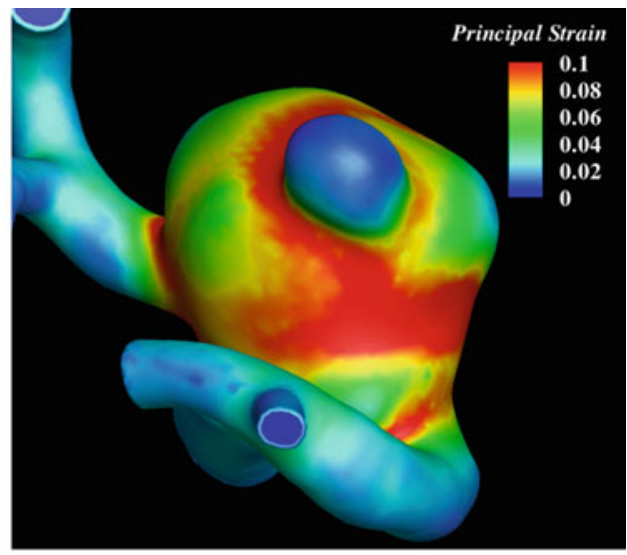

(b) Model 2

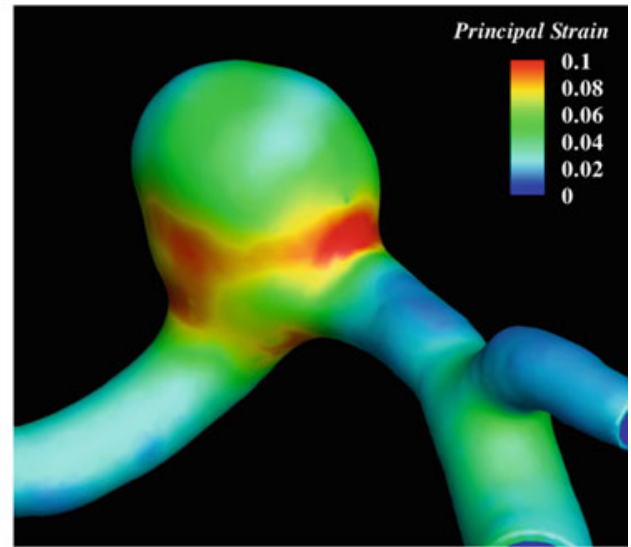

(d) Model 4 stationary domain. In this case, the no-slip boundary conditions mean the blood flow velocity is identically zero at the fluid-solid interface, and the traction compatibility conditions are no longer applicable. The rigid wall assumption is often employed in vascular blood flow simulations due to the significant simplifications and computational cost savings it engenders.

\section{Discrete modeling}

3.1 Mesh generation for vascular fluid-structure interaction

A comprehensive and robust finite element meshing approach for patient-specific arterial geometries coming from medical imaging data, with particular emphasis on cerebral aneurysm configurations, was developed in Zhang et al. (2009). The unique feature of this approach is that the meshes contain both the blood volume and the solid arterial wall, which enable the analyst to use three-dimensional solids to model the behavior of the arterial wall. The fluid-solid meshes are also compatible at their interface, which significantly sim- plifies analysis. The reader is referred to Zhang et al. (2009) for the details of the mesh generation methodology.

The meshing techniques developed in Zhang et al. (2009) are applied to four patient-specific cerebral aneurysm models, shown in Fig. 1. In all cases, the middle cerebral artery (MCA) bifurcation is considered, and the inflow and outflow branches are labeled in the figure. Models 2 and 3 correspond to the ruptured cases, while Models 1 and 4 came from unruptured aneurysms. We would like to note that in Model 4 , the location of the aneurysm is not exactly at the MCA bifurcation but rather downstream of it. We would also like to note the bleb feature at the tip of the aneurysm dome in Model 2. Blebs are indicative of excessive wall stretching, and aneurysms with blebs are considered to be at high risk of rupture.

The four models are used in vascular fluid-structure interaction analysis presented later in the article. In all cases, long inlet and outlet branches are included in the computational models to minimize the effect of inlet and outlet boundary conditions. (See Castro et al. 2006 for the importance of including sufficiently long inlet branch vessels in the computation of cerebral aneurysm flows.) 
Fig. 8 Volume-rendered blood flow velocity magnitude near peak systole $(t=0.08 \mathrm{~s})$

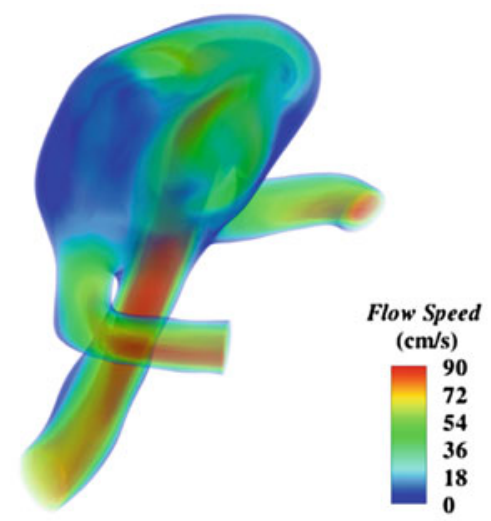

(a) Model 1 - rigid wall

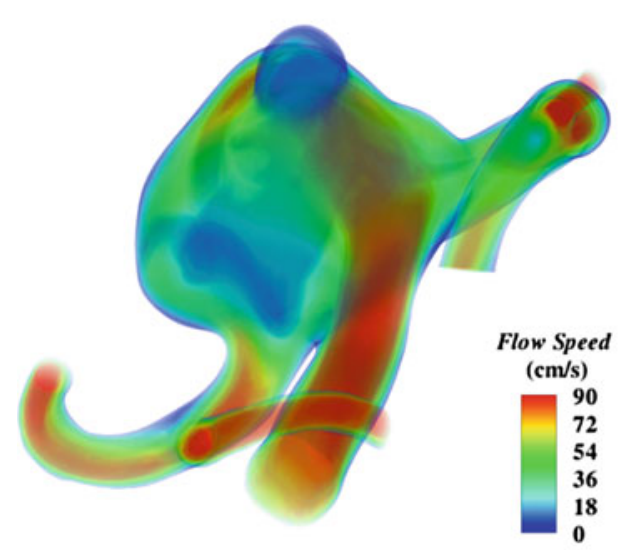

(c) Model 2 - rigid wall

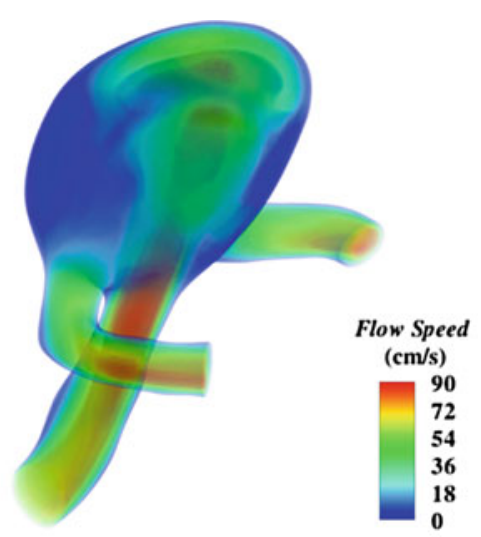

(b) Model 1 - flexible wall

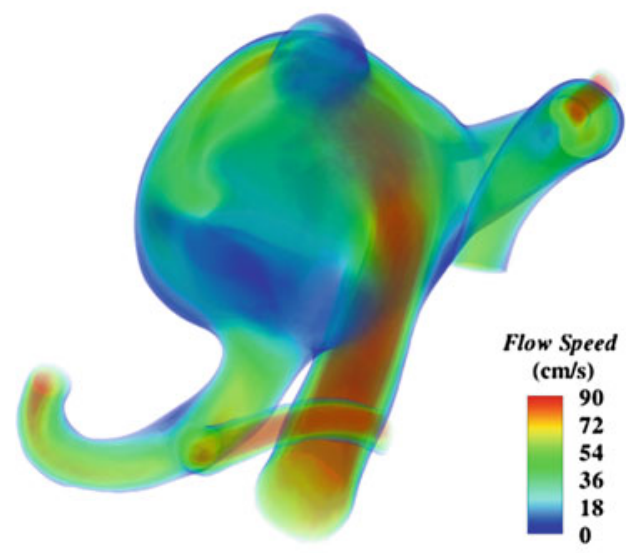

(d) Model 2 - flexible wall
The meshes for both fluid and solid regions consist of linear tetrahedral elements, and the solid wall is meshed using two layers of elements in the through-thickness direction. The choice of tetrahedral over hexahedral discretization is motivated by the relative simplicity of the former approach with respect to the latter for meshing of complex geometrical configurations. Boundary layer meshing is employed in the fluid region to enhance the resolution of wall quantities, such as the shear stress. Figure 2 zooms on the inlet branch of Model 1 where one can clearly see the solid wall and the high-quality boundary layer fluid mesh. The meshes for the remaining three models are of similar quality.

Mesh sizes for all models are summarized in Table 1. The local element Reynolds number based on the mean blood flow speed during the heart cycle is about 10 in the domain interior and decreases to about 1.5 near the solid wall due to boundary layer meshing. The proposed numerical methodology used for the fluid mechanics problem, discussed in the sequel, is known to deliver accurate prediction of flow phenomena for this range of Reynolds numbers, on both tetrahedral and hexahedral discretizations. On a related note, in a recent study (Takizawa et al. 2010b), the authors showed that the quantities of hemodynamic interest, such as the wall shear stress, are accurately represented for cerebral aneurysm flows at the level of mesh resolution employed in this work.

\subsection{Discretization and solution strategies}

The solid and fluid mesh motion equations are discretized using the Galerkin approach. The fluid formulation makes use of the recently proposed residual-based variational multiscale method (Bazilevs et al. 2007). The residual-based variational multiscale methodology is built on the theory of stabilized and multiscale methods (see Brooks and Hughes 1982 for an early reference; Hughes et al. 2004 for a comprehensive review; and Tezduyar 2003; Bazilevs et al. 2007 for specific expressions employed in the definition of stabilization parameters). The methodology applies equally well to laminar and turbulent flows and is thus attractive for applications where the nature of the flow solution is not known a priori. The time-dependent discrete equations are solved using the generalized- $\alpha$ time integrator proposed in Chung and Hulbert (1993) for the equations of structural mechanics, developed in Jansen et al. (1999) for fluid dynamics, and further extended in Bazilevs et al. (2008) to fluid-structure interaction. A monolithic solution strategy is adopted in 
Fig. 9 Volume-rendered blood flow velocity magnitude near peak systole $(t=0.08 \mathrm{~s})$

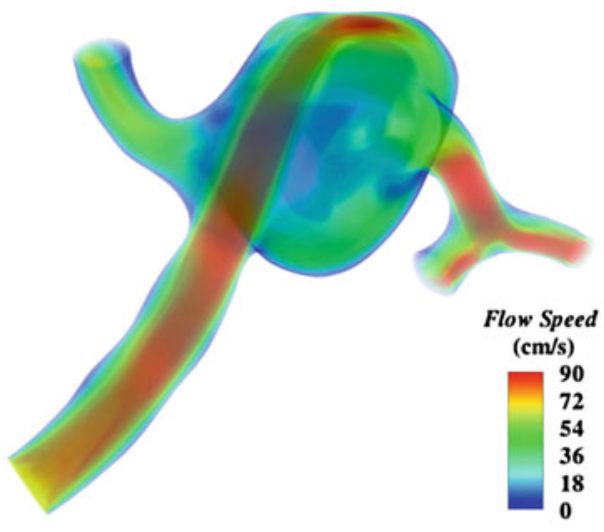

(a) Model 3 - rigid wall

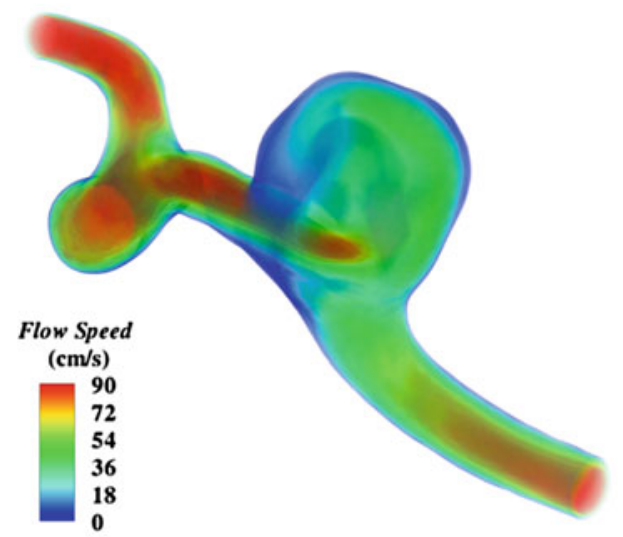

(c) Model 4 - rigid wall

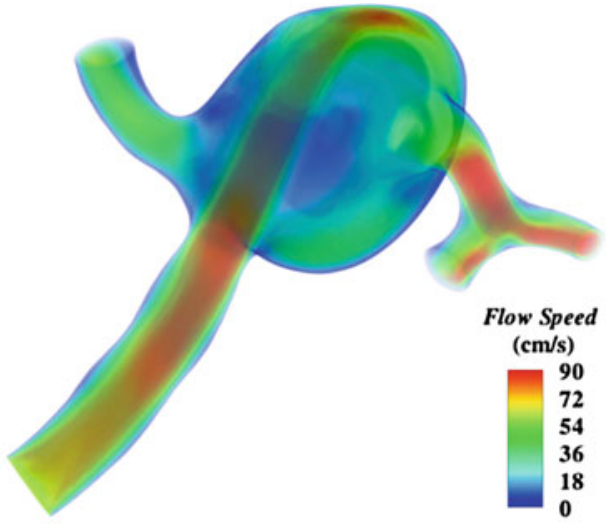

(b) Model 3 - flexible wall

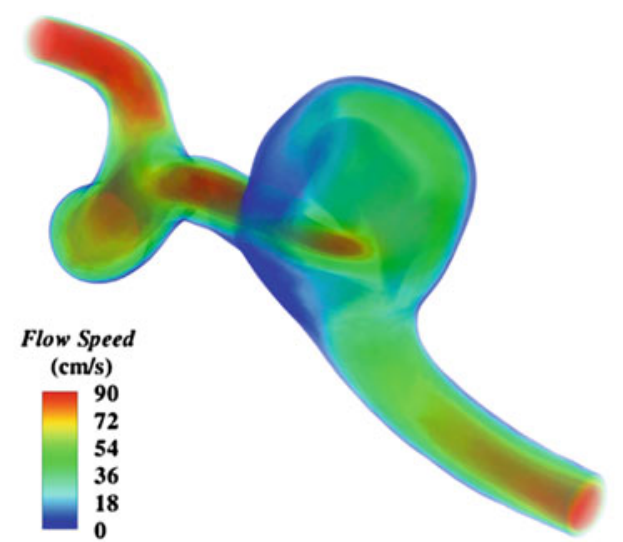

(d) Model 4 - flexible wall which the increments of the fluid, solid, and mesh motion variables are obtained by means of a Newton-Raphson procedure in a simultaneous fashion (see Bazilevs et al. 2006, 2008 for details). The effect of the mesh motion on the fluid equations is omitted from the tangent matrix for efficiency, as advocated in Bazilevs et al. (2009a).

\subsection{Boundary conditions}

Time-varying velocity boundary conditions are applied at the inlet branch in the fluid subdomain. We aim to simulate several cases whose inlet cross-sectional areas can vary significantly from one model to another (see Table 2). This variation is due to the use of patient-specific data and is quite natural in practice. For the four models considered in this work, shown in Fig. 1, the ratio of the largest to smallest inlet cross-sectional area exceeds a factor of two. In this case, an attempt to impose the same volumetric flowrate as a function of time for all four models will result in the inflow velocity variation that is over a factor of two between the patients, which is not physiological. Instead, we chose to impose the same area-averaged inflow velocity for all models, which is more realistic. Figure 3 shows the inlet velocity as a func- tion of time during the heart cycle used as an inlet boundary condition for all models. These data were taken from Isaksen et al. (2008).

Resistance boundary conditions are set at the outlet branches in the fluid subdomain. The resistance boundary conditions are of the form

$p=C_{r} q+p_{0}$,

where $q$ is the volumetric flowrate, $C_{r}$ is a resistance constant, and $p_{0}$ is the ambient pressure level selected such that the pressure fluctuates between 80 and $120 \mathrm{mmHg}$ during the heart cycle.

At all inlets and outlets, the solid and mesh movement boundary conditions are

$\boldsymbol{u} \cdot \boldsymbol{n}^{s}=0$,

$\boldsymbol{\sigma}^{s} \boldsymbol{n}^{s}-\left(\boldsymbol{n}^{s} \cdot \boldsymbol{\sigma}^{s} \boldsymbol{n}^{s}\right) \boldsymbol{n}^{s}=0$,

which allows a given arterial branch to slide in its cut plane, but precludes it from penetrating it. This boundary condition gives more realistic arterial wall displacement patterns than fixed inlet and outlet cross sections. The solid wall is also 
Fig. 10 Wall shear stress near peak systole $(t=0.08 \mathrm{~s})$

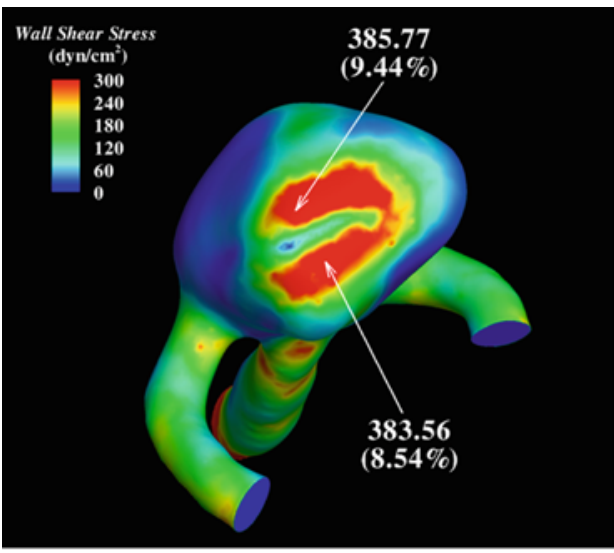

(a) Model 1 - rigid wall

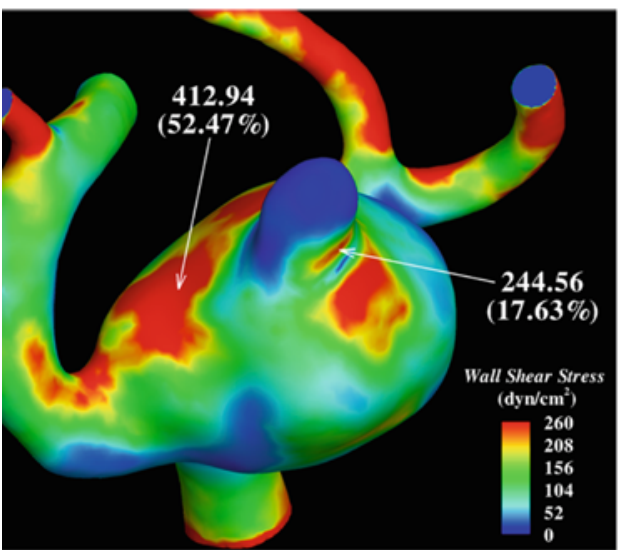

(c) Model 2 - rigid wall

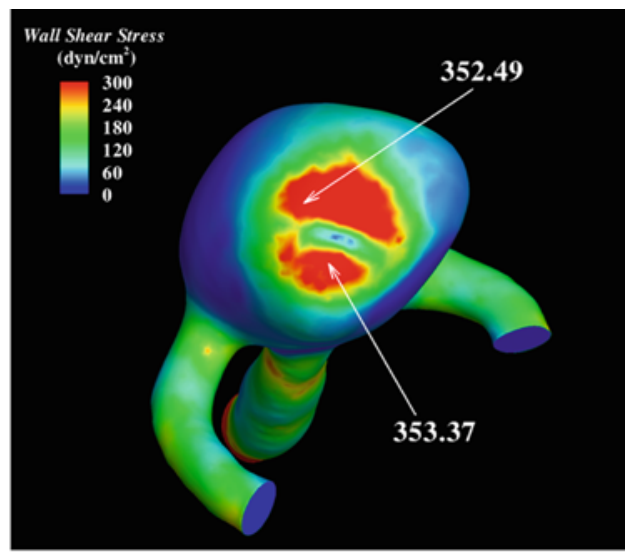

(b) Model 1 - flexible wall

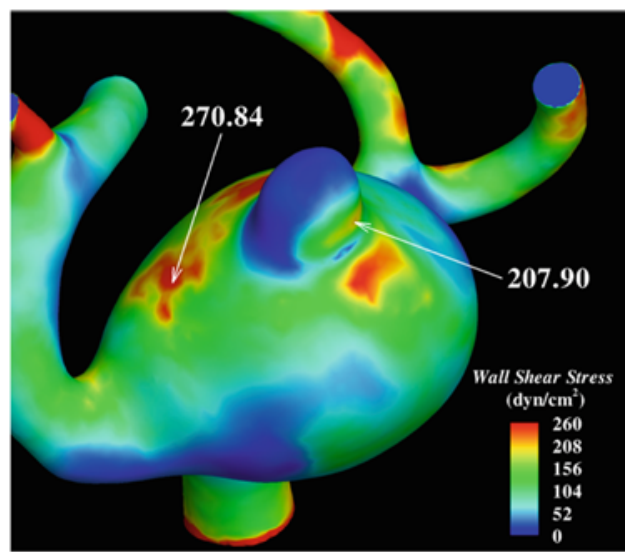

(d) Model 2 - flexible wall subjected to zero traction boundary conditions at the outer surface.

Remark The solid free-slip boundary condition applied at the inlet and outlet surfaces implies that the branches are able to deform in the radial direction. As a result, the inlet and outlet cross-sectional areas change during the heart cycle. This change, which is on the order of $10 \%$ between peak systole and low diastole, is accounted for when comparing rigid and flexible wall simulations as follows. We first compute the flexible wall cases and record the inlet cross-sectional area changes during the heart cycle. We then use this information to scale the inlet velocity for the rigid cases such that, for a given model, the inflow flowrate is the same between the rigid and flexible cases.

\subsection{Material parameters and wall thickness}

In the computations presented in the next section, the density and dynamic viscosity of the fluid are set to $1.0 \mathrm{~g} / \mathrm{cm}^{3}$ and $0.04 \mathrm{~g} /(\mathrm{cms})$, respectively. The density, Young's modulus, and Poisson's ratio of the arterial wall are set to $1.0 \mathrm{~g} / \mathrm{cm}^{3}$, $10^{7} \mathrm{dyn} / \mathrm{cm}^{2}$, and 0.45 , respectively. The material parame- ters employed are identical to those in Isaksen et al. (2008). Note that the choice of units is non-standard (although not uncommon in computational blood flow literature). However, these units are preferred for computer implementation because they lead to better conditioned discrete equations that are easier to solve.

Variable wall thickness is incorporated in the modeling as follows. We assume that the wall thickness at the inlet and outlet branches is $20 \%$ of their effective radii. The effective radius is defined as the radius of the circle that has the same area as a given inlet or outlet. The wall thickness for the remainder of the model is constructed by performing a smooth Laplace operator-based extension of the inlet and outlet thickness data into the domain interior. This vessel wall thickness reconstruction procedure was originally proposed and employed in the simulations of the total cavopulmonary connection in Bazilevs et al. (2009b). The resultant wall thickness distribution for the four models is shown in Fig. 4. While the branch vessel wall thickness is accurately represented, it is felt that the aneurysm dome thickness is somewhat over-estimated. Nevertheless, the resultant dome and branch vessel thickness falls well within the range of values reported for cerebral aneurysms (see, e.g., reference 
Fig. 11 Wall shear stress near peak systole $(t=0.08 \mathrm{~s})$

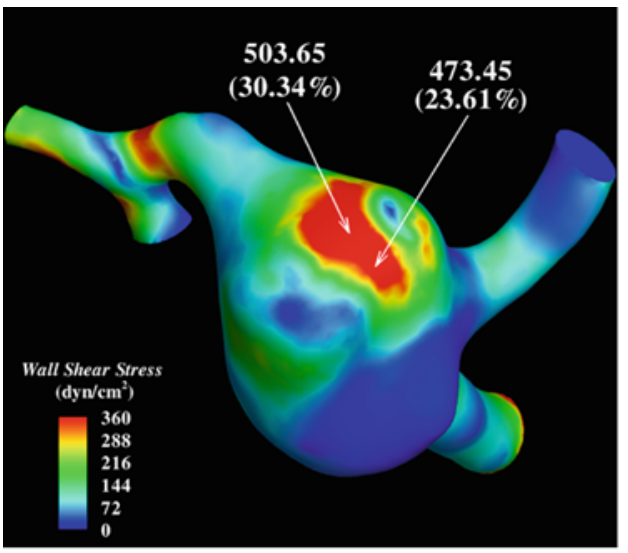

(a) Model 3 - rigid wall

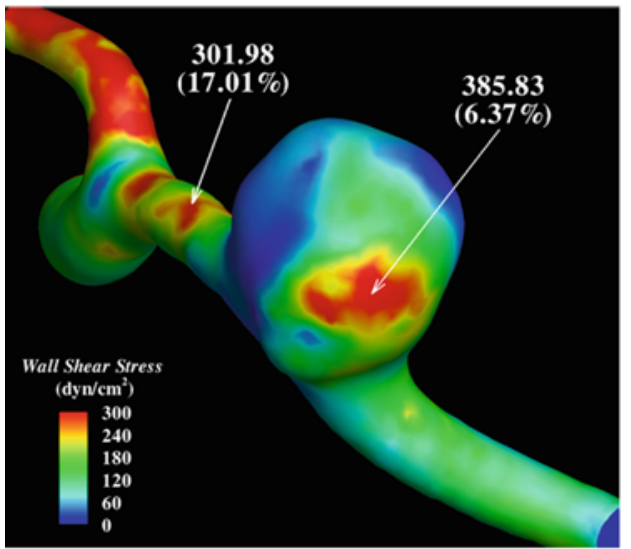

(c) Model 4 - rigid wall

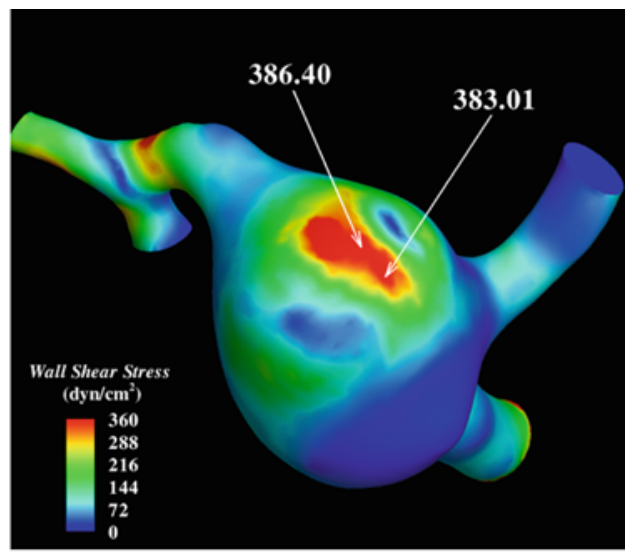

(b) Model 3 - flexible wall

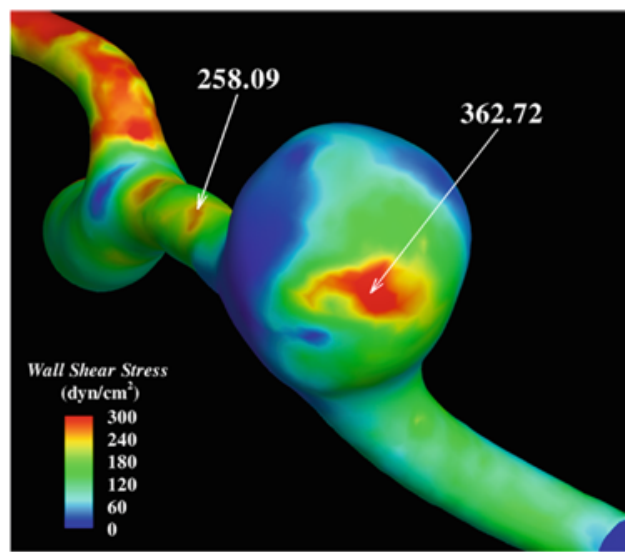

(d) Model 4 - flexible wall
Ryu et al. (2009) where the authors employed high-resolution MRI to obtain aneurysm wall thickness data.).

\subsection{Extraction of wall tension}

The wall tension is associated with aneurysm rupture and merits a close investigation (see, e.g., Isaksen et al. 2008). Aneurysm walls are typically very thin and the largest stresses act in the in-plane directions. As a result, the natural quantity of interest is the principal in-plane stress, which we take for a definition of wall tension. We compute the wall tension as follows. Given the displacement field, we first compute the second Piola-Kirchhoff stress tensor from Eq. (8) and transform it to the Cauchy stress using Eq. (19). The Cauchy stress is rotated to the local coordinate system on every boundary element face as

$\boldsymbol{\sigma}^{s, l}=\boldsymbol{R}^{T} \boldsymbol{\sigma}^{s} \boldsymbol{R}$.

The rotation matrix $\boldsymbol{R}$ takes the form

$\boldsymbol{R}=\left[\begin{array}{ccc}\uparrow & \uparrow & \uparrow \\ \boldsymbol{t}_{1}^{s} & \boldsymbol{t}_{2}^{S} & \boldsymbol{n}^{s} \\ \downarrow & \downarrow & \downarrow\end{array}\right]$, where $\boldsymbol{n}^{s}$, as before, is the outward unit normal, and $\boldsymbol{t}_{1}^{s}$ and $\boldsymbol{t}_{2}^{s}$ are the two orthogonal tangent vectors on the outer surface of the solid. Having rotated the stress tensor to the local coordinate system, we modify it by directly imposing zero traction boundary conditions on the appropriate components of $\boldsymbol{\sigma}^{s, l}$, namely

$\sigma_{3 i}^{s, l}=\sigma_{i 3}^{s, l}=0 \quad \forall i=1,2,3$.

The eigenvalues of the resultant stress tensor can be computed by solving an appropriate quadratic equation. The wall tension is defined as the largest absolute eigenvalue, which also corresponds to the first principal in-pane stress.

Remark It should be noted that in the fully continuous setting, the zero normal stress boundary condition holds pointwise. This obviates the need to employ (25). However, in the discrete setting, the zero normal stress boundary condition only holds weakly. As a result, the exact point-wise satisfaction of this boundary condition is not guaranteed. The above procedure overwrites the computed values of the normal stress with their exact counterparts. This is often done in structural computations to enhance the accuracy of the 
Fig. 12 Oscillatory shear index (OSI)

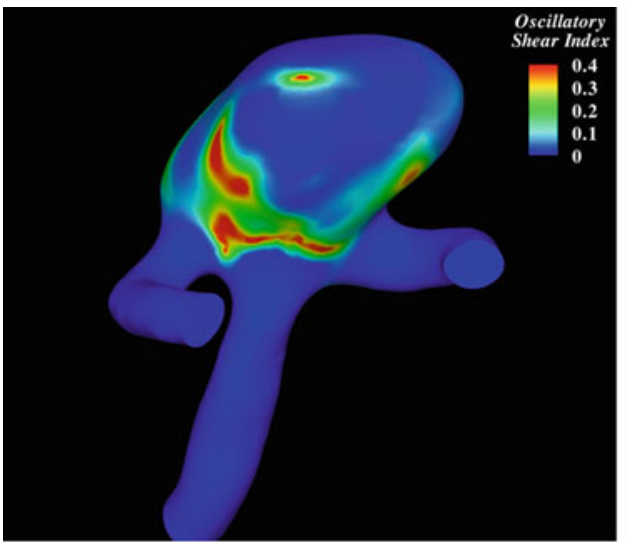

(a) Model 1 - rigid wall

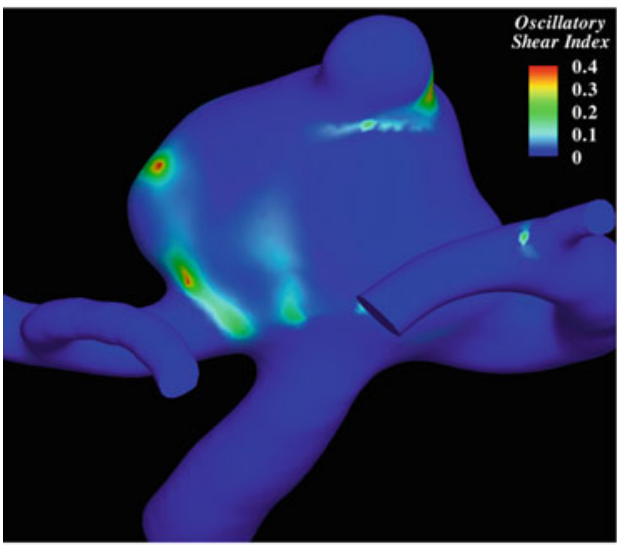

(c) Model 2 - rigid wall

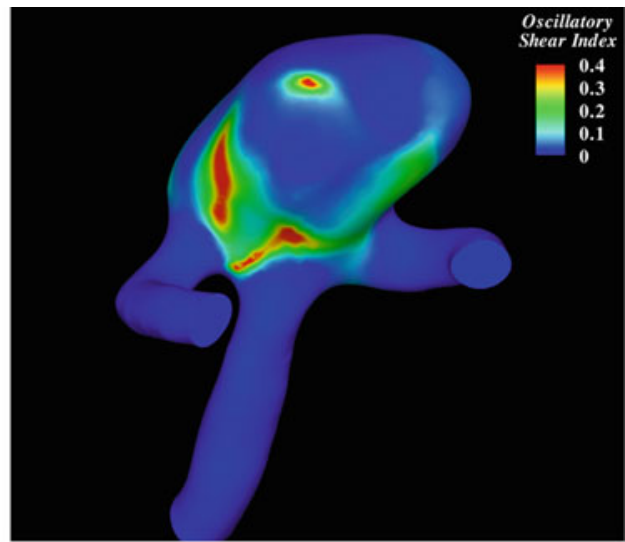

(b) Model 1 - flexible wall

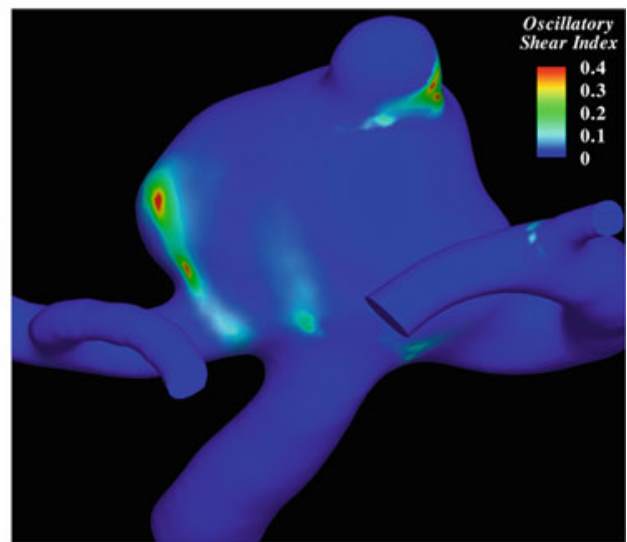

(d) Model 2 - flexible wall computed stress fields (see, e.g., Rank et al. 2005; Hughes et al. 2005).

\section{Computational results}

In this section, we present computational results for the four models we analyzed.

The prestress distribution (see Sect. 2.2.3) over the aneurysm outer surface is shown in Fig. 5. The stresses and their variations are largest in the aneurysm dome for all models. High stress levels tend to occur near the regions of increased curvature.

In Fig. 6, the models are superposed in the configurations corresponding to low diastole and peak systole for better visualization of the relative displacement results. The relative displacement predicted is quite modest and is in good agreement with the observed vessel motions during aneurysm surgery in the clinical practice of one of the authors and predicted in computations by other researchers (see, e.g., Tezduyar et al. 2007; Torii et al. 2008, 2009).
Figure 7 shows a distribution of principal in-plane strain (Green-Lagrange strain measure is employed) at peak systole. At this time instant in the cardiac cycle, the blood pressure is near its peak and, as a result, the strains are also near maximum. The largest strains occur in the aneurysm dome and are on the order of $10-15 \%$. This level of strain suggests that large-strain solid formulations should be employed when simulating fluid-structure interaction phenomena in cerebral aneurysms. However, the strains are not large enough for the stresses to be significantly affected by a specific type of a material non-linearity. As a result, the material model described in this paper is felt to be adequate for the application. We note that in the bleb region of Model 2, the strain levels are quite low considering blebs are indicative of regions of overstretched tissue. Modeling may be enhanced by imposing localized tissue thinning in the bleb areas to capture this effect. However, guidance from histological studies is needed to prescribe appropriate wall tissue thickness in these locations.

Figures 8 and 9 show a comparison of blood flow speed at peak systole for the rigid and flexible wall simulations. In all cases, the flow is complex, with several vortical features 
Fig. 13 Oscillatory shear index (OSI)

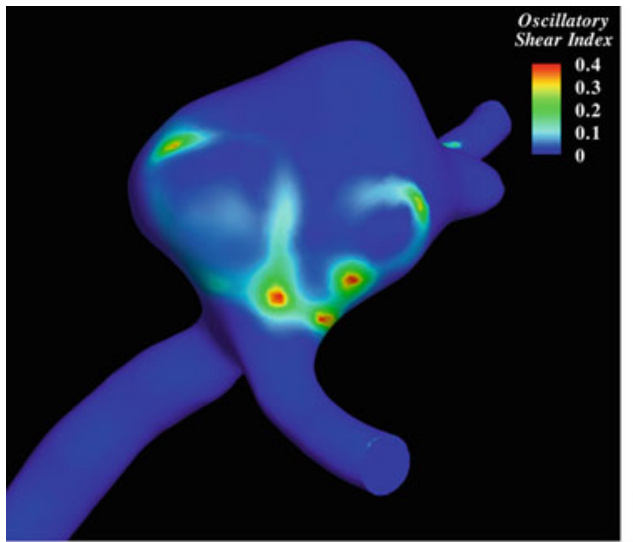

(a) Model 3 - rigid wall

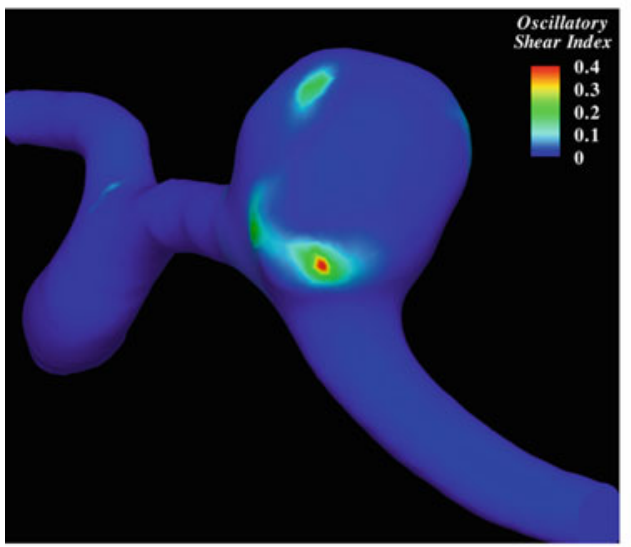

(c) Model 4 - rigid wall

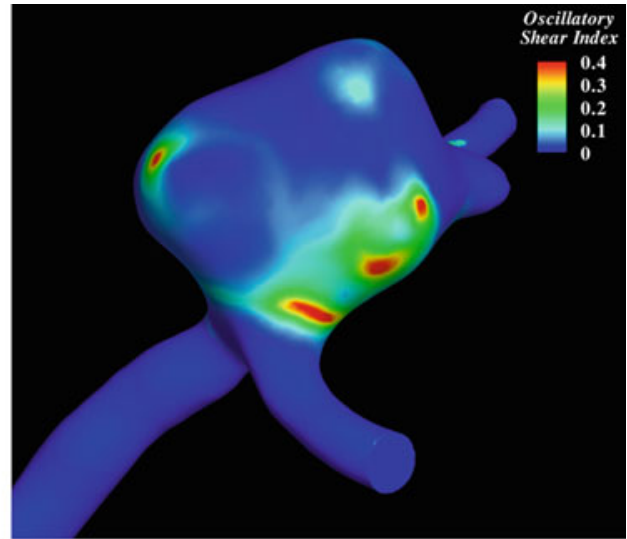

(b) Model 3 - flexible wall

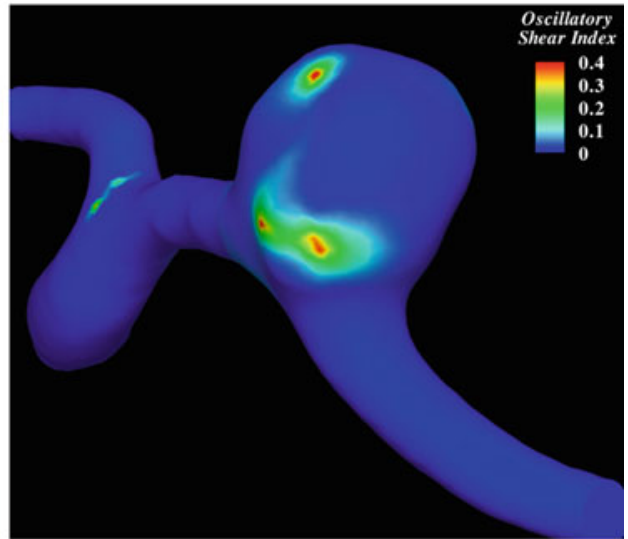

(d) Model 4 - flexible wall present. However, no turbulence is observed in any of the cases. Comparison of rigid and flexible simulations shows the most deviation for Models 1 and 2. In the case of Models 3 and 4, the blood flow velocity results between the flexible and rigid simulations are very similar.

Figures 10 and 11 show the wall shear stress at the fluidsolid interface at the peak inflow flowrate. The wall shear stress in the aneurysm dome is at its maximum near the region where the jet of blood coming from the inflow impinges on the aneurysm wall. Comparisons of the wall shear stress between the rigid and flexible wall cases are also shown in the figures. In all cases, the rigid wall assumption produces an over-estimate of the wall shear stress with respect to the flexible wall computations. Furthermore, the degree to which the wall shear stress is over-predicted is a strong function of the patient-specific geometry. Models 1 and 4 show an overprediction of the WSS in the dome region by less than $10 \%$. In both cases, the flow impingement occurred normal to the aneurysm wall. In the case of Models 2 and 3, for which impingement of the blood flow occurred in the direction tangential to the wall surface, the difference between rigid and flexible wall simulation is more dramatic (52 and $30 \%$ over- estimation for Model 2 and Model 3, respectively.) The spatial distribution of WSS for rigid and flexible wall simulations is likewise different. The differences were most pronounced for Models 1 and 2. The bleb-like feature in Model 2 gave rise to an oscillating flow in its wake and, as a result, produced a complex distribution of the WSS with high spatial variation in this part of the aneurysm dome. Some of this variation is mitigated for the flexible wall case, which gave a significantly more "diffuse" distribution of WSS.

The OSI comparison is shown in Figs. 12 and 13. OSI measures the extent to which the WSS oscillates during the heart cycle. The computation of OSI involves the time average of the wall shear stress that needs to be appropriately defined for moving domain simulations. We employ the method proposed in Takizawa et al. (2010b) where the components of the WSS vector are averaged in the co-rotational coordinate system (i.e., in the coordinate system that rotates with the material). The OSI is highest in the aneurysm dome in all cases. It is also highly localized in a small number of locations on the dome. The localization of OSI occurs in the regions of low mean WSS. The spatial OSI distribution differs between rigid and flexible simulations, the most notable 
Fig. 14 Wall tension near low diastole $(t=0.0 \mathrm{~s})$

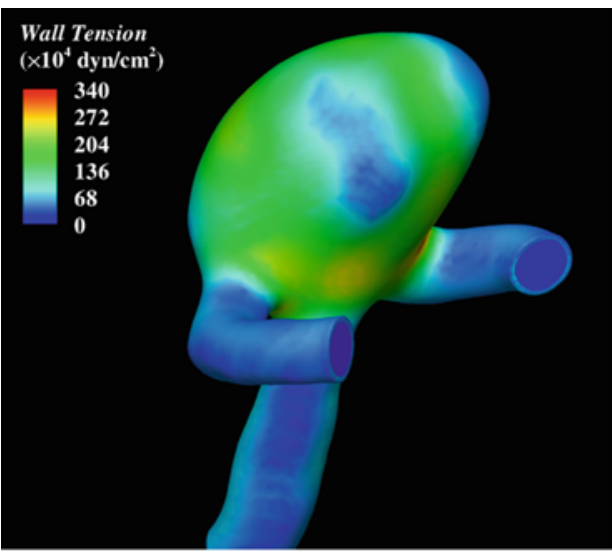

(a) Model 1

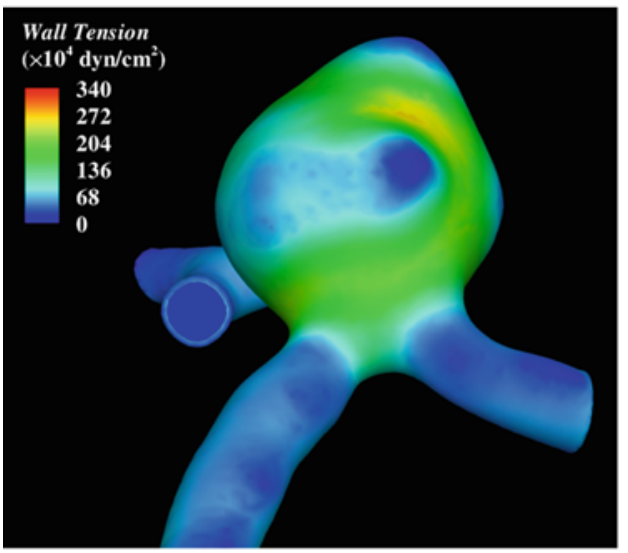

(c) Model 3

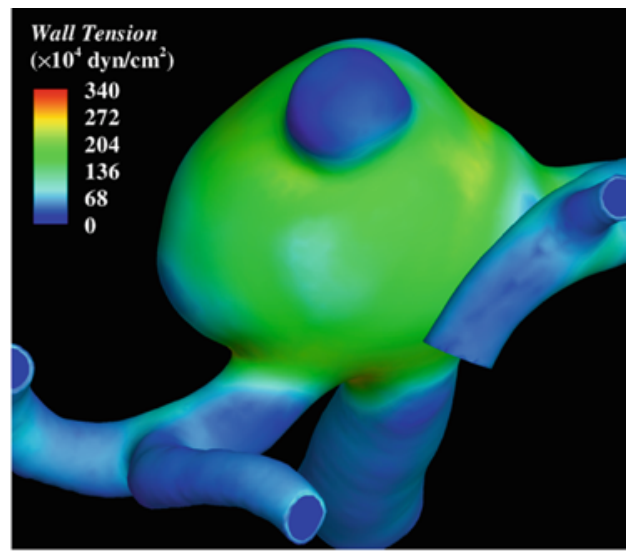

(b) Model 2

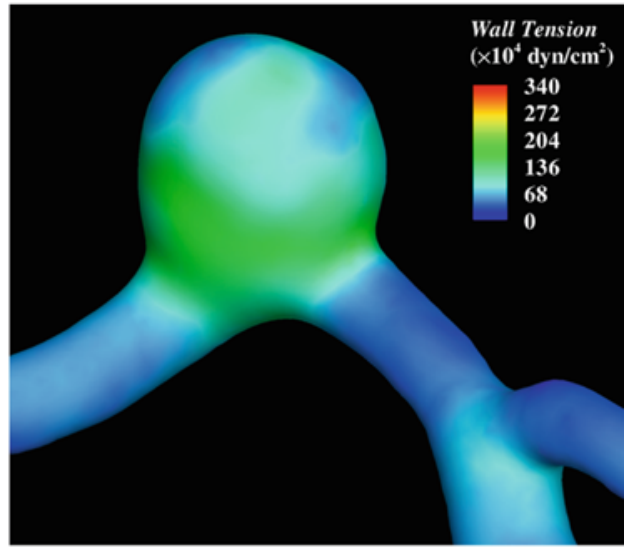

(d) Model 4 differences occurring in Models 1 and 3. In contrast to the WSS, higher levels of OSI are predicted in the flexible wall simulation, which is attributable to the vessel wall motion.

The wall tension results are shown in Figs. 14 and 15. Figure 14 corresponds to peak systole, while Fig. 15 corresponds to low diastole. The magnitude of the wall tension varies through the heart cycle due to the time-dependent nature of the flow. However, the relative wall tension distribution does not vary significantly during the heart cycle. This is apparent from the figures. The wall tension is highest in the aneurysm dome, and not in the inlet and outlet branches. The bands of high wall tension are formed near the aneurysm neck as well as around bleb-like features, which are regions of high surface curvature. Models 2 and 3 exhibited higher wall tension and its spatial variation than Models 1 and 4. As we mentioned previously, Models 2 and 3 correspond to the ruptured aneurysm cases. Model 1 has high wall tension concentrated near the aneurysm neck. However, the wall tension magnitude in the dome is not as high as that in Models 2 and 3. Model 4 gave the overall lowest wall tension magnitude and spatial variability. We would also like to note that in the case of Model 4, the aneurysm dome has a very smooth, almost perfectly spherical shape, and its location is not exactly at the MCA bifurcation, but rather downstream of it.

\section{Conclusions and discussion}

We presented a computational framework for the simulation of vascular fluid-structure interaction using patient-specific models. We added two new features to our modeling framework: (i) We developed a formulation that allows us to compute the vessel wall tissue prestress and use it in the simulations; (ii) We released the constraint of fixing the inlet and outlet branches. Instead, we allowed the branches to "slide" in their cut planes. Both features added to the physical realism of the presented simulations.

The methodology was applied to four patient-specific models of cerebral aneurysms. Two of the models corresponded to ruptured and two to unruptured cases. Besides focusing on the computation of hemodynamics quantities of interest, such as wall shear stress and wall tension distribution for the four patient-specific cases, we also assess the relevance of including flexible wall modeling in the simulations. 
Fig. 15 Wall tension near peak systole $(t=0.08 \mathrm{~s})$

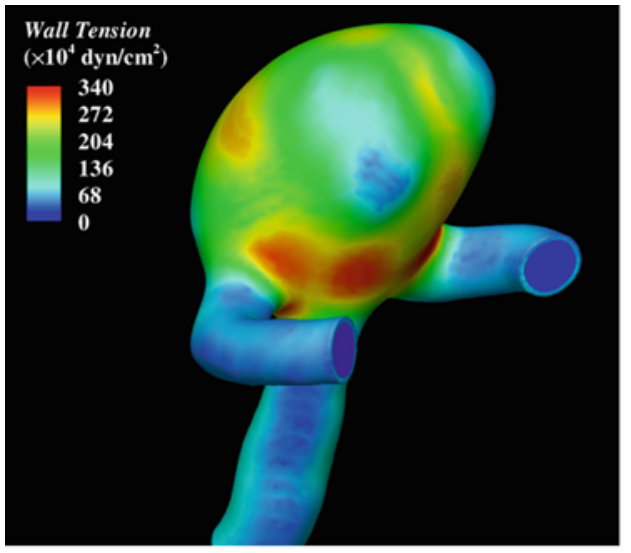

(a) Model 1

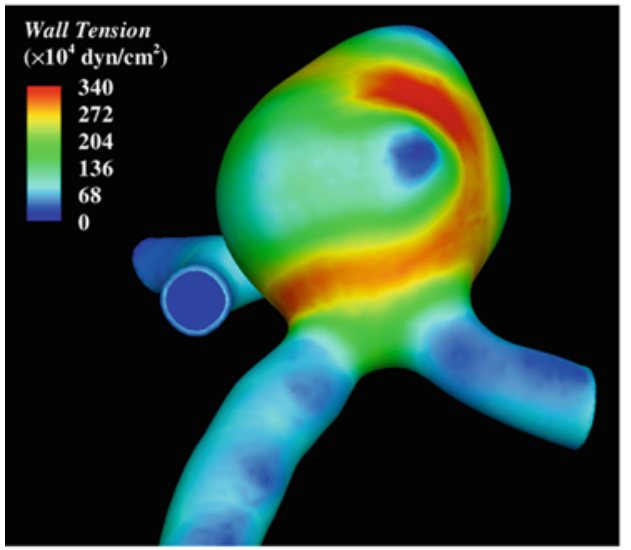

(c) Model 3

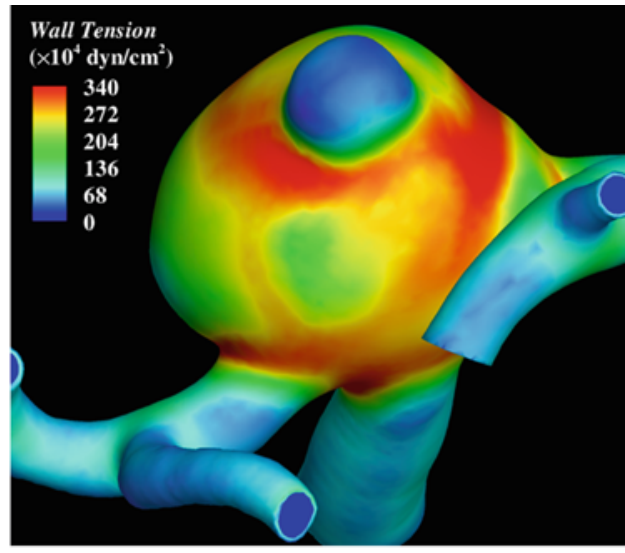

(b) Model 2

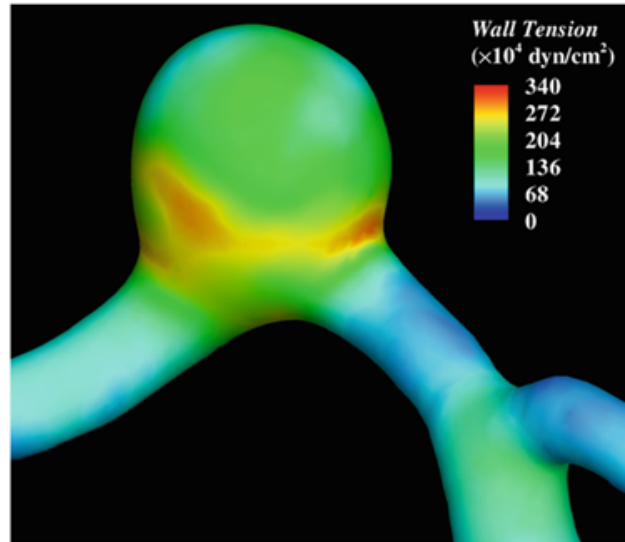

(d) Model 4
The strain levels predicted in the simulations (reaching a maximum of $10-15 \%$ at peak systole) are sufficiently high to necessitate the use of large deformation theory for tissue modeling. However, the strains are not sufficiently high to be in the regime of strong material non-linearity.

The results show that the interaction between the blood flow and wall deformation significantly alters the hemodynamic forces acting on the arterial wall, with respect to rigid wall modeling. Rigid wall simulations consistently overestimated the wall shear stress magnitude, on one case by as much as $52 \%$, which, in our opinion is significant, given the importance of this quantity of interest. In two of the cases, the gross features of the wall shear stress distribution on the arterial wall were significantly different for the rigid and flexible simulations. Rigid versus flexible wall simulation results reinforce the importance of using fluid-structure interaction in patient-specific modeling of cerebral aneurysms. Further observations show that the magnitude of the wall shear stress is a strong function of the inlet branch orientation and the angle of impingement of the blood on the arterial wall.

Pathophysiologically, abnormal levels of WSS and OSI degrade the vessel wall and initiate aneurysm formation, and biomechanically, aneurysm rupture occurs when wall ten- sion exceeds the wall tissue strength. The present simulation shows that WSS, OSI and wall tension are highly dependent on the three-dimensional conformation of the lesion. In the unruptured cases (Models 1 and 4), wall tension was relatively low and relatively evenly distributed in the aneurysm dome. In the ruptured cases (Models 2 and 3), wall tension was higher and distributed in a ring-formed shape around bleb-like formations on the dome. Model 1, the unruptured case, showed relatively high wall tension magnitude (and variation) compared to Model 4, but not to the extent of Models 2 and 3.

A recent article Watton et al. (2009) identified another hemodynamic quantity that affects the growth and remodeling of aneurysms, which is the wall shear stress gradient (WSSG). WSSG measures the spatial variation of the WSS in the vessel wall in-plane directions. In Watton et al. (2009), the in-plane gradient was easily obtainable due to the simple geometry of the vessel employed. However, WSSG requires an appropriate definition in the case of complex patient-specific geometry.

The presences of localized blebs are known to be associated with rupture. This might indicate that rupture occurred because of the strength of the wall tissue was exceeded by 
the wall tension, leaving overstretched tissue remnants at the rupture site. If reproduced in larger clinical series, this proposed computational method might be evolved to a tool for better prediction of future rupture risk. As a result, in the future, we plan to perform simulations of more patient-specific models so as to enhance our understanding of the underlying phenomena and their relationship to clinically observed events.

The wall thickness is very hard or impossible to obtain experimentally, so we plan to make use of the geometry data (such as local branch radii) and clinical experience of one of the co-authors to incorporate a reasonable wall thickness of the aneurysm dome and the surrounding branch vessels in the simulations. Recent advances in high resolution MRI for cerebral aneurysms Ryu et al. (2009) leave us hopeful that in the near future, blood vessel wall thickness may be obtained through imaging, thus removing this uncertainty and burden from the modeling.

We would like to note that the structure of arterial tissue generally changes abruptly in the neck region of an aneurysm from healthy tissue with an intact medial layer within the parent artery to thin collagenous tissue in the aneurysm. As a result, homogeneous isotropic material modeling that is employed in this work may lead to inaccurate predictions of the deformation field and thus inaccurate hemodynamic solutions. A possible remedy for this will be to model healthy arterial tissue in the parent branches and collagenous tissue in the aneurysm dome. We hope to pursue this in future studies.

Another advantage of FSI over the rigid wall assumption is that it enables the simulation of a complete mechanical environment of the arterial wall, including the cells within, and, more importantly, its coupling to the hemodynamics. For instance, cyclic stretching may play a significant role with regards to functionality (e.g., gene expression or structural alignment) of endothelial cells (Cummins et al. 2007), smooth muscles, and fibroblasts. This will, in turn, change the elastic properties of the arterial tissue and alter the response of the coupled system.

Acknowledgments We wish to thank the Texas Advanced Computing Center (TACC) at the University of Texas at Austin for providing HPC resources that have contributed to the research results reported within this paper. This work was partially supported by a research grant from the regional health authorities in northern Norway. Support of Teragrid Grant No. MCAD7S032 is also gratefully acknowledged. We thank Prof. Tor Ingebrigtsen, Institute for Clinical Medicine, University of Troms $\varnothing$, Norway, and the Department of Neurosurgery, the University Hospital of North Norway for generously devoting his time to discuss and evaluate the results of this work and their relevance to clinical practice.

Open Access This article is distributed under the terms of the Creative Commons Attribution Noncommercial License which permits any noncommercial use, distribution, and reproduction in any medium, provided the original author(s) and source are credited.

\section{References}

Appanaboyina S, Mut F, Löhner R, Putman C, Cebral J (2009) Simulation of intracranial aneurysm stenting: techniques and challenges. Comput Methods Appl Mech Eng 198:3567-3582

Bazilevs Y, Calo VM, Zhang Y, Hughes TJR (2006) Isogeometric fluidstructure interaction analysis with applications to arterial blood flow. Comput Mech 38:310-322

Bazilevs Y, Calo VM, Cottrel JA, Hughes TJR, Reali A, Scovazzi G (2007) Variational multiscale residual-based turbulence modeling for large eddy simulation of incompressible flows. Comput Methods Appl Mech Eng 197:173-201

Bazilevs Y, Calo VM, Hughes TJR, Zhang Y (2008) Isogeometric fluidstructure interaction: theory, algorithms, and computations. Comput Mech 43:3-37

Bazilevs Y, Gohean JR, Hughes TJR, Moser RD, Zhang Y (2009a) Patient-specific isogeometric fluid-structure interaction analysis of thoracic aortic blood flow due to implantation of the Jarvik 2000 left ventricular assist device. Comput Methods Appl Mech Eng 198:3534-3550

Bazilevs Y, Hsu M-C, Benson DJ, Sankaran S, Marsden AL (2009b) Computational fluid-structure interaction: methods and application to a total cavopulmonary connection. Comput Mech 45:77-89

Brooks AN, Hughes TJR (1982) Streamline upwind/Petrov-Galerkin formulations for convection dominated flows with particular emphasis on the incompressible Navier-Stokes equations. Comput Methods Appl Mech Eng 32:199-259

Castro MA, Putman CM, Cebral JR (2006) Computational fluid dynamics modeling of intracranial aneurysms: effects of parent artery segmentation on intra-aneurysmal hemodynamics. Am J Neuroradiol 27:1703-1709

Cebral JR, Castro MA, Appanaboyina S, Putman CM, Millan D, Frangi AF (2005) Efficient pipeline for image-based patient-specific analysis of cerebral aneurysm hemodynamics: technique and sensitivity. IEEE Trans Med Imaging 24:457-467

Chung J, Hulbert GM (1993) A time integration algorithm for structural dynamics with improved numerical dissipation: the generalized- $\alpha$ method. J Appl Mech 60:371-375

Cummins PM, von Offenberg Sweeney N, Killeen MT, Birney YA, Redmond EM, Cahill PA (2007) Cyclic strain-mediated matrix metalloproteinase regulation within the vascular endothelium: a force to be reckoned with. Am J Physiol Heart Circ Physiol 292:H28-H42

Fernández MA, Gerbeau J-F, Gloria A, Vidrascu M (2008) A partitioned Newton method for the interaction of a fluid and a 3D shell structure. Technical Report RR-6623, INRIA

Figueroa CA, Vignon-Clementel IE, Jansen KE, Hughes TJR, Taylor CA (2006) A coupled momentum method for modeling blood flow in three-dimensional deformable arteries. Comput Methods Appl Mech Eng 195:5685-5706

Figueroa CA, Baek S, Taylor CA, Humphrey JD (2009) A computational framework for fluid-solid-growth modeling in cardiovascular simulations. Comput Methods Appl Mech Eng 198:3583-3602

Formaggia L, Gerbeau JF, Nobile F, Quarteroni A (2001) On the coupling of 3D and 1D navier-stokes equations for flow problems in compliant vessels. Comput Methods Appl Mech Eng 191:561-582

Gerbeau J-F, Vidrascu M, Frey P (2005) Fluid-structure interaction in blood flows on geometries based on medical imaging. Comput Struct 83:155-165

Holzapfel GA (2000) Nonlinear solid mechanics, a continuum approach for engineering. Wiley, Chichester

Hughes TJR, Scovazzi G, Franca LP (2004) Multiscale and stabilized methods. In: Stein E, de Borst R, Hughes TJR (eds) Encyclopedia of computational mechanics, vol 3, Fluids, chapter 2. Wiley 
Hughes TJR, Cottrell JA, Bazilevs Y (2005) Isogeometric analysis: CAD finite elements, NURBS exact geometry, and mesh refinement. Comput Methods Appl Mech Eng 194:4135-4195

Humphrey JD (2002) Cardiovascular solid mechanics, cells, tissues, and organs. Springer, New York

Isaksen JG, Bazilevs Y, Kvamsdal T, Zhang Y, Kaspersen JH, Waterloo K, Romner B, Ingebrigtsen T (2008) Determination of wall tension in cerebral artery aneurysms by numerical simulation. Stroke 39:3172-3178

Jansen KE, Whiting CH, Hulbert GM (1999) A generalized- $\alpha$ method for integrating the filtered navier-stokes equations with a stabilized finite element method. Comput Methods Appl Mech Eng 190:305-319

Lagana K, Dubini G, Migliavacca F, Pietrabissa R, Pennati G, Veneziani A, Quarteroni A (2002) Multiscale modelling as a tool to prescribe realistic boundary conditions for the study of surgical procedures. Biorheology 39:359-364

Lipton S, Evans JA, Bazilevs Y, Elguedj T, Hughes TJR (2009) Robustness of isogeometric structural discretizations under severe mesh distortion. Comput Methods Appl Mech Eng 199:357-373

Marsden AL, Feinstein JA, Taylor CA (2008) A computational framework for derivative-free optimization of cardiovascular geometries. Comput Methods Appl Mech Eng 197:1890-1905

Nobile F (2001) Numerical approximation of fluid-structure interaction problems with application to hemodynamics. Ph.D. thesis, EPFL

Rank E, Düster A, Nübel V, Preusch K, Bruhns OT (2005) High order finite elements for shells. Comput Methods Appl Mech Eng 194:2494-2512

Rissland P, Alemu Y, Einav S, Ricotta J, Bluestein D (2009) Abdominal aortic aneurysm risk of rupture: patient-specific FSI simulations using anisotropic model. J Biomech Eng 131:031001

Ryu C-W, Jahng G-H, Kim E-J, Choi W-S, Yang D-M (2009) High resolution wall and lumen MRI of the middle cerebral arteries at 3 tesla. Cerebrovasc Dis 27:433-442

Scotti CM, Finol EA (2007) Compliant biomechanics of abdominal aortic aneurysms: a fluid-structure interaction study. Comput Struct 85:1097-1113

Sforza DM, Putman CM, Cebral JR (2009) Hemodynamics of cerebral aneurysms. Annu Rev Fluid Mech 41:91-107

Simo JC, Hughes TJR (1998) Computational inelasticity. Springer, New York

Takizawa K, Christopher J, Tezduyar TE, Sathe S (2010a) Space-time finite element computation of arterial fluid-structure interactions with patient-specific data. Commun Numer Methods Eng 26: 101-116

Takizawa K, Moorman C, Wright S, Christopher J, Tezduyar TE (2010b) Wall shear stress calculations in space-time finite element computation of arterial fluid-structure interactions. Comput Mech. Published online, doi:10.1007/s00466-009-0425-0

Taylor CA, Humphrey JD (2009) Open problems in computational vascular biomechanics: hemodynamics and arterial wall mechanics. Comput Methods Appl Mech Eng 198:3514-3523
Taylor CA, Hughes TJR, Zarins CK (1998) Finite element modeling of blood flow in arteries. Comput Methods Appl Mech Eng 158: 155-196

Tezduyar TE (2003) Computation of moving boundaries and interfaces and stabilization parameters. Int J Numer Methods Fluids 43: $555-575$

Tezduyar TE, Sathe S, Cragin T, Nanna B, Conklin BS, Pausewang J, Schwaab M (2007) Modelling of fluid-structure interactions with the space-time finite elements: arterial fluid mechanics. Int $\mathrm{J}$ Numer Methods Fluids 54:901-922

Tezduyar TE, Takizawa K, Moorman C, Wright S, Christopher J (2009) Multiscale sequentially-coupled arterial FSI technique. Comput Mech. doi:10.1007/s00466-009-0423-2

Torii R, Oshima M, Kobayashi T, Takagi K, Tezduyar TE (2006a) Computer modeling of cardiovascular fluid-structure interactions with the deforming-spatial-domain/stabilized space-time formulation. Comput Methods Appl Mech Eng 195:1885-1895

Torii R, Oshima M, Kobayashi T, Takagi K, Tezduyar TE (2006b) Fluid-structure interaction modeling of aneurysmal conditions with high and normal blood pressures. Comput Mech 38:482-490

Torii R, Oshima M, Kobayashi T, Takagi K, Tezduyar TE (2007) Influence of the wall elasticity in patient-specific hemodynamic simulations. Comput Fluids 36:160-168

Torii R, Oshima M, Kobayashi T, Takagi K, Tezduyar TE (2008) Fluidstructure interaction modeling of a patient-specific cerebral aneurysm: influence of structural modeling. Comput Mech 43:151-159

Torii R, Oshima M, Kobayashi T, Takagi K, Tezduyar TE (2009) Fluidstructure interaction modeling of blood flow and cerebral aneurysm: significance of artery and aneurysm shapes. Comput Methods Appl Mech Eng 198:3613-3621

Vignon-Clementel IE, Figueroa CA, Jansen KE, Taylor CA (2006) Outflow boundary conditions for three-dimensional finite element modeling of blood flow and pressure in arteries. Comput Methods Appl Mech Eng 195:3776-3796

Watton PN, Raberger NB, Holzapfel GA, Ventikos Y (2009) Coupling the hemodynamic environment to the evolution of cerebral aneurysms: computational framework and numerical examples. J Biomech Eng 131:101003

Wolters BJBM, Rutten MCM, Schurink GWH, Kose U, de Hart J, van de Vosse FN (2005) A patient-specific computational model of fluid-structure interaction in abdominal aortic aneurysms. Med Eng Phys 27:871-883

Zhang Y, Wang W, Liang X, Bazilevs Y, Hsu M-C, Kvamsdal T, Brekken R, Isaksen JG (2009) High-fidelity tetrahedral mesh generation from medical imaging data for fluid-structure interaction analysis of cerebral aneurysms. Comput Model Eng Sci 42:131150

Zunino P, D’Angelo C, Petrini L, Vergara C, Capelli C, Migliavacca F (2009) Numerical simulation of drug eluting coronary stents: mechanics, fluid dynamics and drug release. Comput Methods Appl Mech Eng 198:3633-3644 